\title{
Discours
}

Revue de linguistique, psycholinguistique et

informatique. A journal of linguistics, psycholinguistics and computational linguistics

$3 \mid 2008$

Varia

\section{Caractéristiques syntaxiques de la parenthèse en latin : linéarisation, délimitation et insertion}

Isabelle Charnavel

\section{OpenEdition}

\section{Journals}

Édition électronique

URL : http://journals.openedition.org/discours/4573

DOI : $10.4000 /$ discours.4573

ISSN : 1963-1723

Éditeur :

Laboratoire LATTICE, Presses universitaires de Caen

\section{Référence électronique}

Isabelle Charnavel, « Caractéristiques syntaxiques de la parenthèse en latin : linéarisation, délimitation et insertion », Discours [En ligne], 3 | 2008, mis en ligne le 11 octobre 2017, consulté le 10 décembre 2020. URL : http://journals.openedition.org/discours/4573; DOI : https://doi.org/10.4000/discours 4573

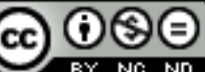

Discours est mis à disposition selon les termes de la licence Creative Commons Attribution - Pas d'Utilisation Commerciale - Pas de Modification 4.0 International. 


\title{
Discours n ${ }^{\circ} 3$
}

\section{«Caractéristiques syntaxiques de la parenthèse en latin : linéarisation, délimitation et insertion »}

\author{
Isabelle Charnavel \\ Ecole Normale Supérieure Ulm \\ University of California, Los Angeles \\ icharnavel@ucla.edu
}

\section{Résumé}

Le but de cet article est d'analyser les règles syntaxiques du placement de l'incidente en latin : l'incidente - ou proposition parenthétique - présente en effet le paradoxe suivant : bien qu'autonome sur un plan structurel (elle forme une proposition indépendante), elle s'insère dans une autre phrase sur un plan syntagmatique (linéairement, elle se situe à l'intérieur d'une autre proposition, l'hôte). Nous proposons que, malgré son autonomie syntaxique, l'incidente obéit à des contraintes syntaxiques précises d'insertion.

Tout d'abord, nous montrons que l'incidente, toujours continue, est délimitée sur ses bords gauche et droit, bien que dépourvue de marqueurs spécifiques : le changement de proposition marque le début de l'incidente, tandis que la reprise de la phrase hôte en montre la fin, au moyen de répétitions pouvant mener à des anacoluthes.

En outre, nous montrons que la place de l'incidente dans la phrase hôte suit des règles spécifiques. Premièrement, les sites d'insertion sont contraints : ils se situent à la frontière des groupes fonctionnels de l'hôte. De plus, il existe des contraintes distributionnelles entre ces sites d'insertion. Nous proposons que le domaine de l'incidente correspond $\mathrm{au}(\mathrm{x})$ constituant(s) sur le(s)quel(s) porte sémantiquement l'incidente et que celle-ci se place toujours à l'intérieur ou à la périphérie de son domaine : une incidente à une proposition indépendante se place en deuxième position de son domaine, tandis qu'une incidente à un constituant occupe la frontière droite de son domaine.

The aim of the paper is to analyse the syntactic rules of the incidental clause in Latin: indeed, the incidental - i.e. parenthetical sentence - exhibits the following paradox: although it is independent from a structural viewpoint (it constitutes a main clause), it is inserted in another sentence from a syntagmatic viewpoint (it linearly occurs inside another sentence, the host). I propose that despite its syntactic autonomy the incidental obeys precise syntactic contraints of insertion.

First of all, I show that the incidental, which is always continuous, is delimited on both boundaries, even if it does not have any specific marker: the clause 
change marks the beginning of the incidental whereas the host start marks its end, by means of repetitions that may lead to anacoluthons.

Furthermore, I argue that the position of the incidental in the host sentence obeys specific rules. First, the insertion spots are constrained: they occur between the functional groups of the host sentence. Moreover, there are distributional contraints to decide which insertion spot to use. I propose that the domain of the incidental consists of the constituent(s) over which the incidental semantically scopes and that it always occurs inside or on the periphery of its domain: the incidental to a root clause occurs at the second position of its domain, whereas the incidental to a phrase is on its right border.

\section{Texte intégral}

1. L'objet de cet article est de décrire et d'analyser les spécificités de l'incidente en latin, en termes de délimitation et d'insertion. Nous choisissons le terme d'incidente pour désigner d'un point de vue spécifiquement syntaxique ce qui est généralement appelé parenthèse: l'incidente correspond ici à une proposition qui, bien qu'autonome syntaxiquement, est toutefois insérée dans la chaîne linéaire d'une autre phrase : sa phrase hôte.

2. Il s'agit alors de comprendre comment une proposition qui est indépendante sur un plan structurel peut, sur un plan syntagmatique, être insérée dans une autre phrase. Le problème est d'autant plus criant en latin que les deux critères importants d'identification des parenthèses ne sont pas disponibles : intonation et ponctuation sont absents des textes latins ${ }^{1}$. Toutefois, cela comporte l'avantage de montrer que les incidentes n'obéissent pas seulement à des contraintes discursives, mais suivent également des règles syntaxiques précises de linéarisation ; de fait, même en l'absence de ces signes discursifs, les incidentes peuvent être identifiées.

3. Nous verrons ainsi non seulement que l'incidente, même si elle ne possède pas de marqueurs spécifiques de frontières, peut être indirectement délimitée sur ses bords gauche et droit. De plus, nous montrerons que son placement à l'intérieur de l'hôte obéit à des contraintes spécifiques : ses sites d'insertion sont contraints et leur distribution est gouvernée par des règles précises.

\section{Délimitation de l'incidente}

4. Avant d'analyser la position de l'incidente par rapport à la phrase hôte, il convient d'examiner la manière dont elle est délimitée dans la chaîne linéaire de la phrase.

5. Sa première caractéristique, qui facilite sa délimitation, est sa continuité linéaire: l'incidente n'est jamais discontinue dans la chaîne syntagmatique. A partir de là, sa délimitation est assurée, en grande partie, par ses frontières : il s'agit donc d'analyser dans quelle mesure les termes qui se trouvent sur ses bords gauche et droit fixent les limites de l'incidente.

\footnotetext{
${ }^{1}$ Cette étude est principalement basée sur un corpus de textes cicéroniens comprenant les Correspondances (les cinq premiers livres), et plusieurs discours (Contre Pison, Contre Vatinius, Pour Milon, Pour Sestius).
} 


\subsection{Frontière gauche}

6. On observe trois types de marquage à la frontière gauche de l'incidente :

- les marqueurs, tels que les connecteurs, qui apparaissent prototypiquement en tête d'une phrase racine ;

- les marqueurs de type phrastique distinct du type de la phrase hôte, tels que les mots interrogatifs ou les interjections ;

- tout terme violant une contrainte de précédence linéaire dans la phrase hôte

\subsubsection{Connecteur}

Nombre d'incidentes sont introduites par des connecteurs, comme et ('et'), -que ('et'), atque ('et'), neque ('et...ne...pas'), enim ('en effet'), etenim ('et de fait'), nam ('de fait'), sed ('mais'), autem ('or'), tamen ('cependant'), at ('mais'), ou atqui ('et pourtant'), qui permettent ainsi de marquer le bord gauche de l'incidente :

[1] Cic. Q. Fr. 1, 3, 8 :

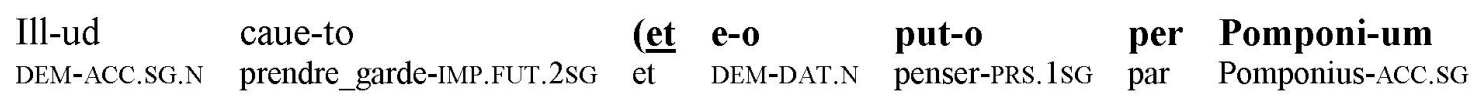

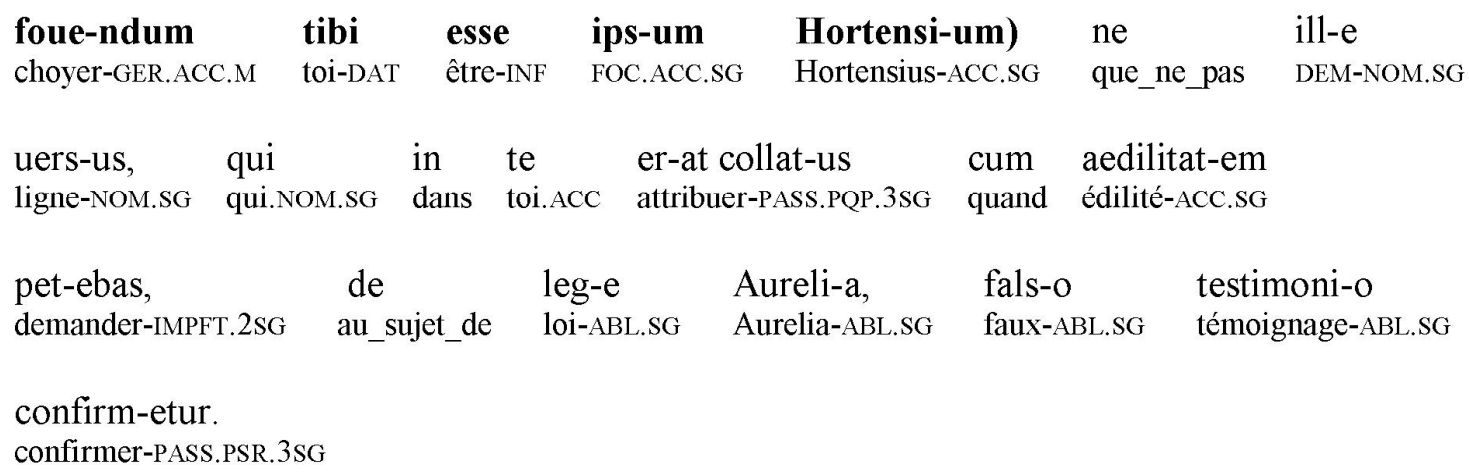

«Il te faut aussi prendre garde (et pour cela tu devras, je crois, par l'intermédiaire de Pomponius, cultiver Hortensius lui-même) qu'un faux témoignage ne vienne confirmer que tu es l'auteur de cette épigramme sur la loi Aurelia qui t'avait été attribuée quand tu briguais l'édilité. » (L.-A. Constans²)

7. Mais il ne s'agit pas là de marqueurs spécifiques, qui signaleraient sans ambiguïté le début d'une incidente : en effet, tous ces connecteurs pourraient relier deux éléments de la phrase hôte, syntagmes nominaux dans certains cas, ou surtout propositions. Toutefois, dans la mesure où ces connecteurs se trouvent le plus souvent à l'initiale d'une nouvelle proposition, ils fonctionnent comme des indices qui attirent l'attention du lecteur ou de l'interlocuteur. C'est ensuite, notamment, la dissemblance syntaxique ou sémantique entre les deux éléments situés de part et d'autre du connecteur, qui permet l'interprétation du segment suivant le connecteur comme incidente.

\footnotetext{
${ }^{2}$ Nous avons délibérément évité d'élaborer des traductions personnelles, afin de ne pas nous exposer au risque d'orienter nos traductions vers ce que nous voulions montrer. Nous avons donc repris les traductions de l'édition des Belles Lettres.
} 


\subsubsection{Adverbe}

Outre les connecteurs, des adverbes, comme ibi ('là'), tum ('alors'), ita ('ainsi') ou sic ('ainsi'), permettent de signaler le début d'une incidente, à condition d'être ana- ou cataphoriques :

[2] Cic. Att. 2, 9, 4 :

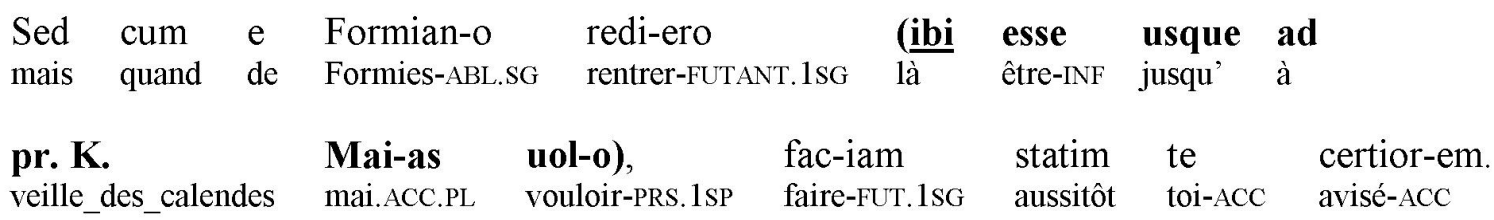

«De toute façon, quand je serai rentré de Formies (je compte y être jusqu'au 30 avril), je t'en aviserai aussitôt. » (L.-A. Constans)

8. Là encore, de tels adverbes pourraient syntaxiquement appartenir à la phrase hôte ; c'est pourquoi l'interprétation comme incidente de la séquence qu'ils introduisent nécessite également d'autres éléments, comme l'apparition d'un verbe principal qui ne soit pas compatible avec la phrase hôte. Cependant, ces adverbes, du fait de la relation endophorique qu'ils instaurent, introduisent indirectement les incidentes, en marquant leur frontière.

\subsubsection{Pronom}

Des pronoms anaphoriques permettent également d'introduire les incidentes :

[3] Cic. Att. 5, 21, 5 :

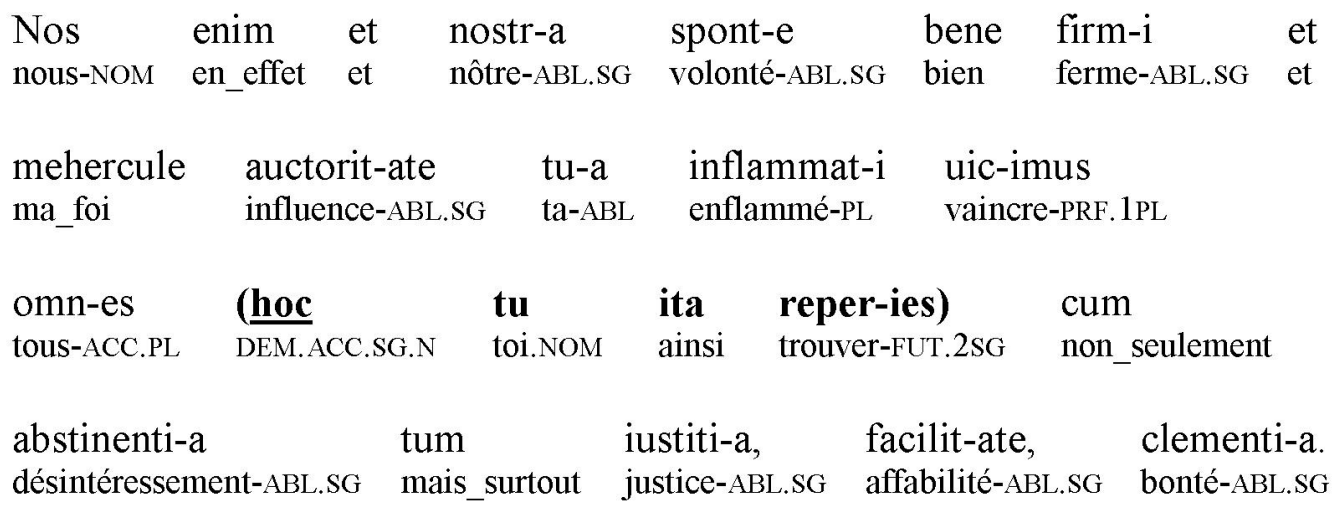

«Une résolution personnelle très ferme et aussi, ma foi, l'aiguillon de tes conseils, m'ont mis au-dessus de tous (tu t'en rendras compte toi-même), non seulement pour le désintéressement, mais pour l'esprit de justice, l'affabilité, la bonté. » (L.-A. Constans et Jean Bayet)

9. Certes, un pronom anaphorique pourrait être interprété comme appartenant à la phrase hôte. Toutefois, dans l'exemple [3], la seule interprétation possible de hoc ('cela') comme élément de la phrase hôte, lors de son apparition dans la chaîne linéaire, serait de le considérer comme un ablatif qui aurait la fonction de circonstant, même si plusieurs autres ablatifs, compléments d'agent, précédent déjà le verbe. Mais dès le deuxième 
terme de l'incidente, $t u$ ('toi'), cette interprétation est invalidée, ce qui fait de hoc ('cela') un indice assez clair de frontière gauche de l'incidente.

\subsubsection{Verbe}

Connecteurs, adverbes, et pronoms peuvent donc marquer le bord gauche de l'incidente. Mais l'apparition, dans la chaîne linéaire, du verbe de l'incidente est souvent nécessaire à son interprétation comme incidente. Il est dès lors logique que le verbe lui-même puisse également correspondre au début de l'incidente :

[4] Cic. Att. 1, 10, 1 :

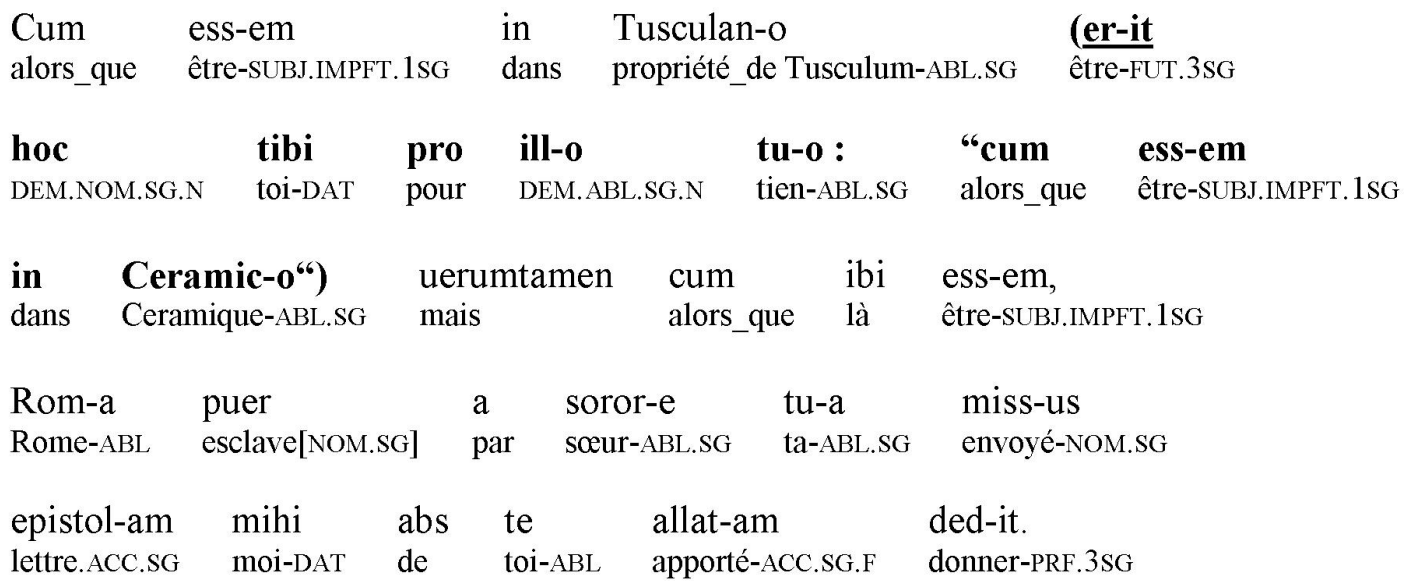

«J'étais dans ma propriété de Tusculum (cette formule est pour répondre à ton 'J'étais au Céramique'), je me trouvais donc là, quand un jeune esclave envoyé de Rome par ta sœur me remit une lettre qu'on avait apportée pour moi de ta part [...]. » (L.-A. Constans)

10. De fait, sa présence même, comme verbe conjugué, suggère l'existence d'une nouvelle proposition.

\subsubsection{Répétition d'un élément de la phrase hôte}

L'incidente est cependant délimitée de manière encore plus nette lorsqu'un élément de la phrase hôte est répété en début d'incidente :

[5] Cic. Fam. 3, 2, 1 (à Ap. Claudius) :

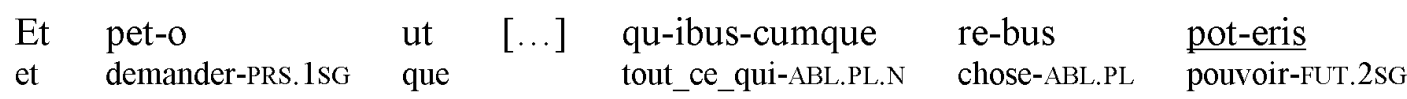

$\begin{array}{lllll}\begin{array}{l}\text { (pot-eris } \\ \text { pouvoir-FUT.2SG }\end{array} & \begin{array}{l}\text { autem } \\ \text { or }\end{array} & \begin{array}{l}\text { plurim-is), } \\ \text { très_nombreux-ABL.PL }\end{array} & \begin{array}{l}\text { prospic-ias } \\ \text { veiller-SUBJ.PRS.2SG }\end{array} & \begin{array}{l}\text { et } \\ \text { et }\end{array} \\ \begin{array}{l}\text { consul-as } \\ \text { prendre_soin-SUBJ.PRS.2SG }\end{array} & \begin{array}{l}\text { ration-ibus } \\ \text { intérêt-DAT.PL me-is. }\end{array} & \\ \end{array}$

«Par tous les moyens en ton pouvoir (et tu n'en manqueras pas), prends soin de mes intérêts. » (L.-A. Constans)

11. C'est surtout pour des raisons sémantiques et pragmatiques que la répétition d'un même terme indique l'apparition d'une incidente : de fait, une telle reprise signale que l'auteur commente l'emploi du terme, qui est alors repris en usage et/ou en mention. Toutefois, 
ce genre de répétitions constitue aussi un indice syntaxique du début de l'incidente. De fait, en latin, deux mêmes termes juxtaposés ne peuvent appartenir à la même proposition que dans des cas très rares : leur juxtaposition signale donc bien plutôt la présence de deux propositions, dont les frontières sont justement constituées par ces termes.

\subsubsection{Terme négatif}

De manière plus étonnante peut-être, la frontière gauche d'une incidente est très souvent constituée par un terme négatif, tel que non ('ne...pas'), neque ('et...ne...pas'), nihil ('rien'), nemo ('personne'), ou numquam ('jamais').

[6] Cic. Att. 1, 16, 8 :

$\begin{array}{llllll}\begin{array}{l}\text { Idem } \\ \text { le_même-NOM.SG }\end{array} & \begin{array}{l}\text { ego } \\ \text { moi-NOM }\end{array} & \begin{array}{l}\text { ille } \\ \text { DEM-NOM.SG.M }\end{array} & \begin{array}{l}\text { (non } \\ \text { ne_pas }\end{array} & \begin{array}{l}\text { enim } \\ \text { en_effet }\end{array} & \begin{array}{l}\text { mihi } \\ \text { moi-DAT }\end{array} \\ \begin{array}{l}\text { uide-or } \\ \text { sembler-PRs.1sG }\end{array} & \begin{array}{l}\text { insolenter } \\ \text { immodérément }\end{array} & \begin{array}{l}\text { gloriari } \\ \text { se_vanter-INF }\end{array} & \text { cum quand } & \begin{array}{l}\text { de } \\ \text { de }\end{array} & \begin{array}{l}\text { me } \\ \text { moi-ABL }\end{array}\end{array}$

\begin{tabular}{|c|c|c|c|c|c|c|}
\hline $\begin{array}{l}\text { apud } \\
\text { auprès_de }\end{array}$ & $\begin{array}{l}\text { te } \\
\text { toi-ACC }\end{array}$ & $\begin{array}{l}\text { loqu-or, } \\
\text { parler-PRs.1sG }\end{array}$ & $\begin{array}{l}\text { in } \\
\text { dans }\end{array}$ & $\begin{array}{l}\text { e-a } \\
\text { DEM.ABL.SG.F }\end{array}$ & $\begin{array}{l}\text { praesertim } \\
\text { surtout }\end{array}$ & \\
\hline & qu-am & nol-o & & ali-is & leg-i) & idem \\
\hline lettre-ABL.SG & REL.ACC. & ne_pas_v & uloir-PRS.1SG & autres-DAT.PL & lire-INF.PASS & le_même-NOM.SG \\
\hline
\end{tabular}

$\begin{array}{llllll}\text { inquam } & \text { ego } & \text { recre-au-i } & \text { adflict-os } & \text { anim-os } & \text { bon-orum } \\ \text { dis-je } & \text { moi.NOM } & \text { ranimer-PFT.1sG } & \text { abattus-ACC.PL } & \text { courage-ACC.PL } & \text { bon-GEN.PL }\end{array}$

un-umqu-emque confirm-ans, excit-ans.

chacun-ACC.SG rassurant-NOM.SG stimulant-NOM.SG

«Ce fut moi encore (car je n'ai pas le sentiment de manquer à la modestie quand je parle de moi devant toi, surtout dans une lettre que je ne veux pas qu'on lise à d'autres), ce fut moi, dis-je, qui rendis courage une fois encore aux bons citoyens, rassurant, stimulant chacun. » (L.-A. Constans)

\subsubsection{Terme interrogatif}

Quand l'incidente est de type interrogatif, c'est un morphème interrogatif qui permet de marquer le bord gauche de l'incidente :

[7] Cic. Att. 1, 13, 4 :

\begin{tabular}{|c|c|c|c|c|c|c|c|}
\hline $\begin{array}{l}\text { Tu-us } \\
\text { ton-NOM }\end{array}$ & $\begin{array}{l}\text { autem } \\
\text { or }\end{array}$ & NOM.SG.M & $\begin{array}{l}\text { c-us } \\
\text { NOM.SG }\end{array}$ & $\begin{array}{l}(\operatorname{scin} \underline{n} \\
\text { sais-tu }\end{array}$ & $\begin{array}{l}\text { qu-em } \\
\text { qui.ACC.SG }\end{array}$ & $\begin{array}{l}\text { dic-am ?), } \\
\text { dire-SUBJ.PRS.1SG }\end{array}$ & $\begin{array}{l}\text { qu-o } \\
\text { qui.ABL.SG }\end{array}$ \\
\hline $\begin{array}{l}\text { u } \\
\text { bi.NOM }\end{array}$ & $\begin{array}{l}\text { me } \\
\text { moi.ACC }\end{array}$ & $\begin{array}{l}\text { scrips-isti, } \\
\text { écrire-PFT.2sG }\end{array}$ & $\begin{array}{l}\text { postea } \\
\text { après }\end{array}$ & $\begin{array}{l}\text { quam } \\
\text { que }\end{array}$ & $\begin{array}{l}\text { non } \\
\text { ne_pas }\end{array}$ & $\begin{array}{l}\text { aud-eret } \\
\text { oser-SUBJ.IMPFT.3sG }\end{array}$ & $\begin{array}{l}\text { reprehend-ere } \\
\text { blâmer-INF }\end{array}$ \\
\hline & commen & nous- & comm & & PRS. $3 \mathrm{SG}$ & $\begin{array}{l}\text { admodum } \\
\text { tout à fait }\end{array}$ & $\begin{array}{l}\text { it } \\
\text { PRS.3SG }\end{array}$ \\
\hline
\end{tabular}

«Ton grand ami, (tu sais qui je veux dire ?), celui dont tu m'as écrit qu'il s'était mis à louer parce qu'il n'osait pas blâmer, a pour moi, si l'on en croit les marques qu'il en donne, beaucoup d'amitié [...]. » (L.-A. Constans) 
12. Ainsi, dans cet exemple, la particule interrogative enclitique -nĕ ('est-ce que'), devenue ici $-n$ par apocope, marque sans ambiguité le début d'une incidente interrogative. En effet, il s'agit là d'un terme qui se situe de manière normale en début de proposition interrogative, et le statut d'incidente ne modifie pas ce fait.

\subsubsection{Interjection}

De manière parallèle, des termes exclamatifs, adjectifs ou pronoms, peuvent marquer le début d'une incidente exclamative, même si cela est plus rare. Des interjections, qui permettent de renforcer le statut exclamatif d'une proposition, se trouvent parfois aussi en début d'incidente :

[8] Cic. Att. 1, 16, 5 :

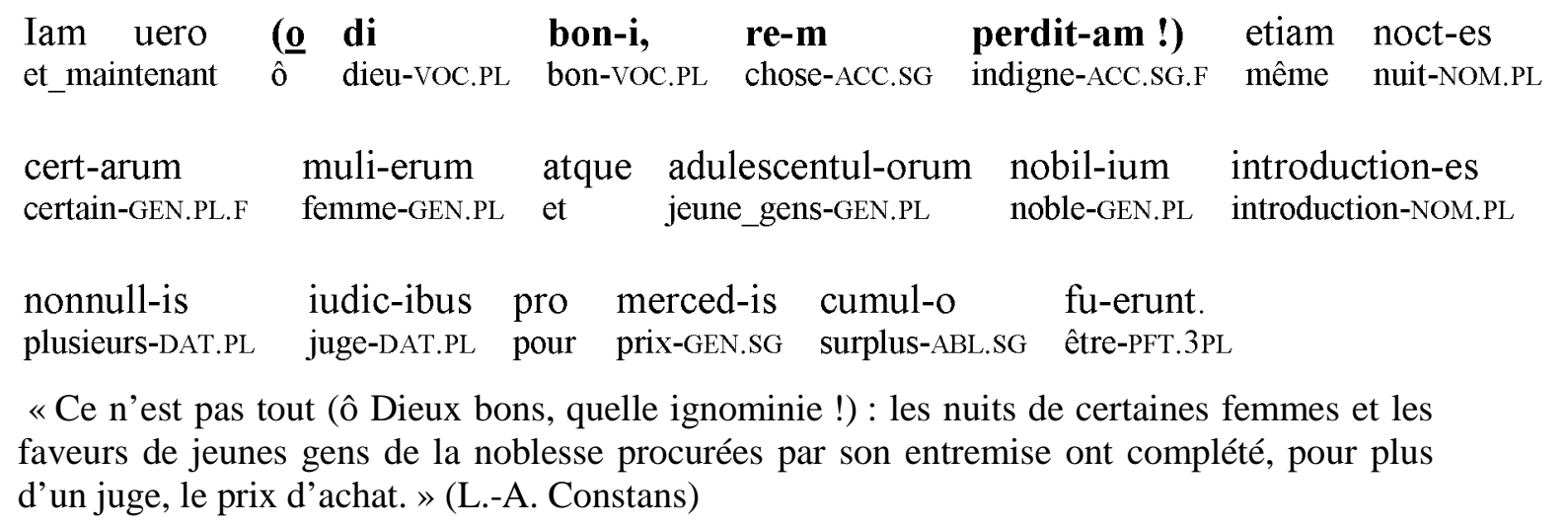

13. De fait, dans cet exemple, l'interjection $o$ signale le passage à un autre registre intonatif et énonciatif, ce qui marque l'entrée dans l'incidente.

14. Au total, on observe que la frontière gauche des incidentes n'est pas marquée de manière spécifique par un élément dont le rôle serait exclusivement celui de marquer le début d'une incidente. Toutefois, on constate que certains éléments apparaissent de manière récurrente sur le bord gauche de l'incidente, ce qui incite à penser que, même s'ils ne sont pas réservés à cette fonction de manière spécifique, ils contribuent à délimiter l'incidente. On peut regrouper ces éléments en trois catégories :

15. - les premiers correspondent à des éléments qui signalent de manière typique le début d'une proposition indépendante ; les connecteurs et les termes interrogatifs en sont les plus clairs représentants. Dans ces cas, c'est donc en tant que proposition indépendante que l'incidente est délimitée.

16. - dans d'autres cas, c'est le changement de proposition qui est marquée en premier lieu ; il s'agit alors davantage d'éléments marquant une rupture que d'éléments constituant une première position de proposition. Les pronoms ou les termes en reprise appartiennent à ce type où l'incidente est alors délimitée, avant tout, en tant que proposition insérée qui interrompt la phrase hôte.

17. - enfin, un troisième groupe rassemble les éléments qui marquent le changement de proposition plutôt sur un plan pragmatique ou énonciatif que syntaxique. C'est par 
exemple le cas des interjections, et sans doute des négations. C'est alors en tant que parenthèse que l'incidente est ici délimitée ${ }^{3}$.

18. Quoi qu'il en soit, l'incidente paraît, dans la grande majorité des cas, être délimitée sur le bord gauche par certains éléments déterminés, même si le marquage reste assez hétérogène. Qu'en est-il du bord droit ?

\subsection{Frontière droite}

Dans la plupart des cas, le dernier terme de l'incidente correspond à son verbe principal :

[9] Cic. Att. 1, 19, 4 :

$\begin{array}{lllllll}\text { Popul-o } & \text { autem } & \text { et } & \text { Pompei-o } & \text { (nam } & \text { id } & \text { quoque } \\ \text { peuple-DAT.SG } & \text { or } & \text { et } & \text { Pompée-DAT } & \text { car } & \text { DEM.ACC.SG.N } & \text { aussi }\end{array}$

uol-ebam) satisfaci-ebam empti-one.

vouloir-IMPFT.1SG satisfaire-IMPFT.1SG achat-ABL.SG

«D'un autre côté, je donne satisfaction au peuple et à Pompée (et c'est ce que je désire aussi) par ma proposition d'achat. » (L.-A. Constans)

19. Il ne s'agit pas là, véritablement, d'un marqueur de fin d'incidente. Il faut plutôt considérer que, dans la mesure où une proposition, en latin, se termine fréquemment par un verbe, il est attendu que l'incidente, en tant que proposition, se comporte de même. Dès lors, le verbe correspond à un marqueur par défaut de la fin de l'incidente.

20. Dans d'autres cas, on constate que le dernier terme de l'incidente correspond au complément de son verbe principal :

[10] Cic. Att. 5, 21, 9 :

\begin{tabular}{|c|c|c|c|c|c|}
\hline $\begin{array}{l}\text { Id-ibus } \\
\text { ide-ABL.PL }\end{array}$ & $\begin{array}{l}\text { Februari-is, } \\
\text { de_février-ABL.PL }\end{array}$ & $\begin{array}{l}\text { qu-o } \\
\text { REL.ABL.SG }\end{array}$ & $\begin{array}{l}\text { h-as } \\
\text { DEM.AC }\end{array}$ & $\begin{array}{l}\text { litter-as } \\
\text { lettre-ACC. }\end{array}$ & \\
\hline $\begin{array}{l}\text { ded-i, } \\
\text { donner-PFT.1sG }\end{array}$ & $\begin{array}{l}\text { for-um } \\
\text { assises-ACC.sG }\end{array}$ & $\begin{array}{l}\text { institu-eram } \\
\text { commencer-PQPFT.1sG }\end{array}$ & $\begin{array}{l}\text { agere } \\
\text { tenir-INF }\end{array}$ & $\begin{array}{l}\text { Laodice-ae } \\
\text { Laodicée-LOC.SG }\end{array}$ & $\begin{array}{l}\text { Cibyratic-um } \\
\text { de_Cibyre-Acc.sG }\end{array}$ \\
\hline $\begin{array}{l}\text { Apame } \\
\text { d'Apame }\end{array}$ & $\begin{array}{l}\mathrm{e} \\
\mathrm{ACC} . \mathrm{SG}\end{array}$ & $\begin{array}{l}\text { Id-ibus } \\
\text { ide-ABL.PL }\end{array}$ & ars-ABL.PL & $\begin{array}{l}\text { ibidem } \\
\text { au_même_endro }\end{array}$ & \\
\hline
\end{tabular}

$\begin{array}{lllll}\begin{array}{l}\text { Synnadens-e, } \\ \text { de_Synnade-ACC.SG }\end{array} & \begin{array}{l}\text { Pamphyli-um } \\ \text { de_Pamphylie-ACC.SG }\end{array} & \begin{array}{l}\text { (tum } \\ \text { alors }\end{array} & \begin{array}{l}\text { Phemi-o } \\ \text { Phemius-DAT.SG }\end{array} & \begin{array}{l}\text { dispici-am } \\ \text { examiner-FuT.1SG }\end{array}\end{array}$

кع́pac), Lycaoni-um, Isauric-um.

flûte de_Lycaonie-ACC.SG d'Isaurie-ACC.SG

«Aujourd'hui 13 février, je commence à tenir mes assises à Laodicée pour les gens de Cibyra et d'Apamée ; à partir du 15 mars, toujours à Laodicée, je jugerai pour Synnade, la Pamphylie (c'est alors que j'aurai l'œil à une flûte pour Phémius), la Lycaonie, l'Isaurie. » (L.-A. Constans et Jean Bayet)

\footnotetext{
${ }^{3}$ En effet, dans notre terminologie, si le terme d'incidente envisage la structure étudiée sur un plan exclusivement syntaxique, celui de parenthèse inclut la perspective pragmatique.
} 
21. Il s'agit là aussi d'un marquage par défaut: dès que le complément obligatoire est donné, l'incidente peut se clore.

22. Dans aucun de ces exemples, toutefois, la frontière droite n'est marquée de manière spécifique ; cela semble être en revanche le cas dans l'exemple suivant :

[11] Cic. Att. 1, 12, 2 :

$\begin{array}{llllll}\begin{array}{l}\text { Libert-um } \\ \text { affranchi-ACC.SG }\end{array} & \text { ego } & \text { habe-o } & \text { sane } & \text { nequam } & \text { homin-em, } \\ \text { moi-NOM } & \text { avoir-PRS.1sG } & \text { tout_à_fait } & \text { vaurien } & \text { homme-ACC.SG }\end{array}$

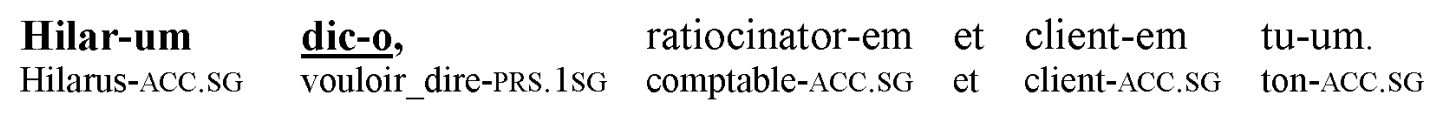

«J'ai un affranchi, un parfait coquin — j'ai nommé Hilarus—, qui est comptable, et ton client. » (L.-A. Constans)

23. Au premier abord, il semble s'agir ici de cas où le verbe de la proposition incidente, dico ('je veux dire'), se place, normalement, en dernière position. Toutefois, on observe que dico apparaît systématiquement à la fin des incidentes qui ont pour fonction d'expliciter un nom. On pourrait donc considérer qu'on a ici affaire à un processus de figement, où dico, de verbe principal, deviendrait une particule enclitique signalant le procédé de dénomination. Par là même, dico deviendrait un marqueur du bord droit de l'incidente. Cette dernière hypothèse, toutefois, ne se vérifie pas dans les incidentes plus longues :

[12] Cic. Att. 4, 16, 8 :

$\begin{array}{lllllll}\text { Itaque } & \text { Caesar-is } & \text { amic-i } & \text { (me } & \text { dic-o } & \text { et } & \text { Oppi-um, } \\ \text { c'est_pourquoi } & \text { César-GEN.SG } & \text { ami-NOM.PL } & \text { moi-ACC.SG } & \text { dire-PRs.1sG } & \text { et } & \text { Oppius-ACC.SG }\end{array}$

$\begin{array}{lllll}\begin{array}{l}\text { disrump-aris } \\ \text { briser-PASS.SUBJ.PRS.2SG }\end{array} & \begin{array}{l}\text { licet) } \\ \text { ettre_permis-PRS.3SG }\end{array} & \text { in } & \text { munument-um } & \text { ill-ud } \\ \text { travaux-ACC.SG } & \text { REL.ACC.SG.N }\end{array}$

$\begin{array}{llllll}\text { qu-od } & \text { tu } & \text { toll-ere } & \text { laud-ibus } & \text { sole-bas, } & {[\ldots]} \\ \text { DEM.ACC.SG.N } & \text { toi-NOM } & \text { élever-INF } & \text { louange-ABL.PL } & \text { avoir_l'habitude-IMPFT.2SG } & \end{array}$

$\begin{array}{lll}\text { contemps-imus } & \text { sexcenti-es } & \text { HS. } \\ \text { dépenser-PFT.1PL } & \text { sesterce-ACC.PL } & 60 \_ \text {millions }\end{array}$

«Aussi les amis de César (c'est Oppius et moi que je veux dire, dusses-tu en crever de dépit), nous avons dépensé sans compter, pour ces travaux que tu portais aux nues, [...] 60 millions de sesterces. » (L.-A. Constans)

24. De fait, dico se place après le premier terme de l'explicitation me ('moi') —car il ne s'agit pas à proprement parler de dénomination ici-, mais l'incidente continue. Au final, il est donc difficile de considérer dico comme un des marqueurs possibles du bord droit des incidentes.

25. On constate plutôt, dans la plupart des cas, que c'est moins la frontière droite de l'incidente qui est marquée que la reprise de la phrase hôte. En effet, dans tous les exemples, la frontière droite, de manière encore plus évidente que la frontière gauche, n'est pas délimitée par un terme précis et spécifique. Mais la fin de l'incidente sur la chaîne syntagmatique est souvent signalée par une reprise marquée de la phrase hôte. 


\subsection{Reprise de la phrase hôte}

\subsubsection{Connecteurs}

De fait, on observe très souvent qu'un terme précis signale sans ambiguitté la reprise de la phrase hôte sur la chaîne linéaire. Chez Cicéron, sed ('mais') apparaît très souvent, mais aussi sed tamen ('mais cependant'), et plus rarement, uerum ('mais'), uerum tamen ('mais cependant'), igitur ('donc') et ergō ('donc').

[13] Cic. Pis. 8, 4 :

$\begin{array}{lllll}\mathrm{Qu}-\mathrm{OS} & \mathrm{Q} . \text { Metell-uS } & \text {-fac-io } & \text { iniuri-am } & \text { fortissim-o } \\ \text { REL.ACC.PL } & \text { Metellus-NOM.SG } & \text { faire-PRS.1SG } & \text { injustice-ACC.SG } & \text { très-courageux-DAT.SG }\end{array}$

\begin{tabular}{|c|c|c|c|c|c|}
\hline $\begin{array}{l}\text { uir-o } \\
\text { homme-DAT.SG }\end{array}$ & $\begin{array}{l}\text { mortu-o, } \\
\text { mort.DAT.SG }\end{array}$ & $\begin{array}{l}\mathbf{q u}-\mathbf{i} \\
\text { REL.NOM.SG }\end{array}$ & $\begin{array}{l}\text { ill-um, } \\
\text { DEM.ACC.SG.M }\end{array}$ & $\begin{array}{l}\text { cuius } \\
\text { REL.GEN.SG }\end{array}$ & $\begin{array}{l}\text { pauc-os } \\
\text { peu- } A C C . P L\end{array}$ \\
\hline
\end{tabular}

$\begin{array}{llllll}\text { par-is } & \text { haec } & \text { ciuitas } & \text { tul-it } & \text { cum } & \text { hac } \\ \text { égal-ACC.PL } & \text { DEM.NOM.SG.F } & \text { cité-NOM.SG } & \text { comporter-PFT.3SG } & \text { avec } & \text { DEM.ABL.SG.F }\end{array}$

$\begin{array}{llllll}\begin{array}{l}\text { importun-a } \\ \text { brutal-ABL.SG }\end{array} & \begin{array}{l}\text { belu-a } \\ \text { animal-ABL.SG }\end{array} & \begin{array}{l}\text { confer-am- } \\ \text { comparer-SUBJ.PRS.1SG }\end{array} & \underline{\text { sed }} & \text { ille } & \text { designat-us } \\ \text { mais } & \text { DEM.NOM.SG.M } & \text { désigné-NOM.SG }\end{array}$

$\begin{array}{lllll}\text { consul, } & {[\ldots]} & \begin{array}{l}\text { priuat-us } \\ \text { privé-NOM.SG }\end{array} & \text { fi-eri } & \text { se_produire-INF } \\ \text { consul-[NOM.SG] } & & \text { interdire-PFT.3sG }\end{array}$

«Ces jeux dont Quintus Métellus (j'insulte à la mémoire d'un si grand homme en le comparant, lui qui a trouvé si peu d'égaux dans notre cité, à cet animal sauvage), ces jeux, dis-je, dont, simple consul désigné, il interdit à titre privé la célébration [...]. » (Pierre Grimal)

26. On peut supposer que ces connecteurs représentent des marqueurs spécifiques de reprise après incidente, dans la mesure où leur présence ne se justifie que de cette façon. Certes, dans la langue latine, ces connecteurs ne sont pas réservés à ce rôle, mais dans ce cas précis, leur seule fonction, aussi bien sur le plan syntaxique que sémantique, est de reprendre la phrase hôte après l'incidente. En effet, manifestement, ces connecteurs n'assurent pas, ici, de coordination entre les deux fragments de la phrase hôte : dans l'exemple [13], sed ('mais'), dans le cadre de la phrase hôte, sépare deux syntagmes nominaux; or, étant donné que ceux-ci ont même référence $-Q$. Métellus-, il est difficile, aussi bien sémantiquement que syntaxiquement, de considérer que sed correspond ici à une conjonction de coordination qui relierait ces deux syntagmes; il s'agit plutôt de ce qu'on appelle traditionnellement une apposition, où la présence d'une conjonction de coordination n'est pas requise, d'autant plus, dans ce cas précis, qu'une relation d'opposition entre les deux groupes ne ferait pas sens; cela signifie que la phrase hôte, après suppression de l'incidente, serait au moins étrange sémantiquement, voire agrammaticale. De ce point de vue, on peut d'ailleurs considérer qu'il existe ainsi une certaine dépendance syntaxique de la phrase hôte par rapport à l'incidente, dans la mesure où la présence de sed dans la phrase hôte n'est syntaxiquement correcte qu'en présence de l'incidente. Il s'agit là d'une limite à la suppressibilité de l'incidente que l'on pourrait inférer de son autonomie structurelle. 
27. A partir de là, il est donc légitime de supposer que l'apparition de sed provient de la présence de l'incidente qui précède la particule. S'agit-il alors de coordination entre l'incidente et la phrase hôte ? Manifestement, une telle hypothèse serait difficile à tenir. En effet, cela impliquerait que l'incidente et la phrase hôte se situent sur un même plan structurel, correspondant alors, dans l'exemple [13], à deux phrases principales coordonnées. Mais à ce moment-là, la présence du segment quos Q. Metellus ('ceux-là Quintus Metellus') serait difficilement explicable. En outre, les connecteurs situés sur le bord gauche de l'incidente ne fonctionnent pas comme coordonnants. Dès lors, une coordination sur le bord droit créerait une asymétrie structurelle, source d'agrammaticalité : l'incidente ne peut être insérée sur sa frontière gauche, et coordonnée sur sa frontière droite. Il paraît donc plus correct de considérer que l'incidente est insérée dans la phrase hôte sans aucune coordination de part et d'autre, et que sed, dès lors, ne détient pas de rôle coordonnant, mais correspond à un connecteur adverbial. Cela semble être également le cas des autres connecteurs tamen ('cependant'), uerum ('mais'), ergo ('donc') et igitur ('donc').

28. On peut toutefois se demander en quoi leur sémantisme les détermine à signaler, dans ces cas précis, la reprise d'une phrase hôte après incidente. Car tous les connecteurs ne jouent pas ce rôle, ce qui semble suggérer que le sémantisme spécifique de ces connecteurs-là les y prédispose. Or ces cinq connecteurs semblent se partager en deux groupes principaux : les uns, sed ('mais'), sed tamen ('mais cependant'), uerum ('mais') et uerumtamen ('mais cependant'), sont généralement classés parmi les connecteurs adversatifs, tandis que les autres, igitur ('donc') et ergo ('donc'), appartiennent aux connecteurs consécutifs. Une hypothèse serait alors de penser que ce sémantisme serait approprié d'un point de vue pragmatique. Dans le premier cas, on opposerait l'énonciation de la phrase hôte à celle de l'incidente ('j'ai dit cela, mais je reviens à mon propos'), comme c'est plus clairement le cas dans des expressions du type sed redeamus $a d . . .($ 'mais revenons à...'). Dans le deuxième cas, on voudrait conclure l'énonciation du premier segment de la phrase hôte. Le sémantisme de ces connecteurs se comprendrait donc sur un plan énonciatif, en se rapportant, dans un cas, à l'incidente, dans l'autre cas, au premier segment de la phrase hôte.

29. Mais quoi qu'il en soit, même si l'on préfère considérer que ces connecteurs n'ont d'autre signification, dans ces exemples, que de signaler la reprise de la phrase hôte, il n'en reste pas moins qu'ils fonctionnent bien comme marqueurs structurels dans la chaîne syntagmatique, laissant une trace formelle de la présence de l'incidente à l'intérieur de la phrase hôte.

\subsubsection{Répétition d'un terme de $\mathrm{H} 1^{4}$}

Mais la présence du connecteur n'est pas la seule perturbation syntaxique que l'incidente entraîne dans la phrase hôte. Bien plus souvent, un ou plusieurs termes du premier fragment de la phrase hôte sont repris après l'incidente, ce qui assure la reprise de la phrase hôte sur la chaine syntagmatique ${ }^{5}$. Cela montre d'autant mieux que la suppression de l'incidente n'est guère possible dans ces exemples, ce qui indique à nouveau une

\footnotetext{
${ }^{4} \mathrm{H} 1$ désigne la partie de la phrase hôte qui précède l'incidente.

${ }^{5}$ En termes stylistiques, on peut parler d'antanaclase à propos de cette répétition d'une partie de H1. Voir Pétillon-Boucheron: 2002, p. 207.
} 
certaine dépendance syntaxique, sur le plan de la linéarisation, de la phrase hôte par rapport à l'incidente.

30. En règle générale, c'est le premier terme de la phrase hôte qui est repris, lorsqu'il correspond à un terme grammatical - comme une conjonction de subordination ou une préposition - qui assure la structure de la phrase. Mais la reprise peut aussi correspondre à un résumé du premier segment de la phrase hôte : cela correspond aux phénomènes de reprise dans la langue orale, où le locuteur reprend le début de son propos afin de le remettre en mémoire chez son interlocuteur, voire chez lui-même. Dès lors, on assiste, en quelque sorte, à un redéploiement, sur l'axe syntagmatique, de deux variantes offertes par anaphore - par l'axe paradigmatique. Cela est d'autant plus frappant quand il s'agit vraiment d'une variante sémantique :

[14] Cic. Fin. 4, 56 :

$\begin{array}{llllll}\text { Postea } & \text { tu-us } & \text { ille } & \text { Poenul-us } & \text {-sc-is } & \text { enim } \\ \text { après } & \text { ton-NOM.SG } & \text { DEM.NOM.SG.M } & \text { Phénicien-NOM.SG } & \text { savoir-PRS.2sG } & \text { en_effet }\end{array}$

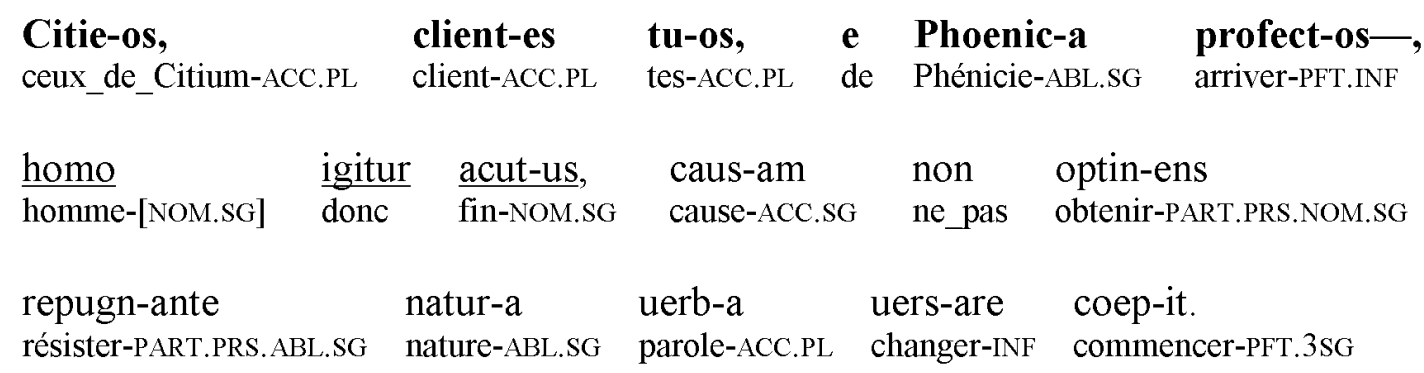

«Alors votre Phénicien (car vous savez que ceux de Citium, vos clients, sont originaires de Phénicie), voyant qu'il ne pouvait gagner sa cause, parce que la nature même luttait contre lui, commença à donner un nouveau tour à ses expressions. » (M. Guyau)

31. Ainsi, dans cet exemple, sur un plan syntaxique, le groupe sujet est repris par igitur ('donc') après l'incidente, tandis que sur un plan sémantique, le référent est désigné autrement. Si tuus ille Poenulus ('votre Phénicien') et homo acutus ('homme fin') appartiennent bien au même paradigme -étant donné que la présence de igitur ('donc'), qui signale la reprise, empêche de considérer homo acutus comme une apposition-, ces deux syntagmes nominaux correspondent à des variantes sémantiques qui peuvent se déployer sur l'axe syntagmatique.

32. En outre, ces reprises sont, bien souvent, accompagnées de inquam ('dis-je'), parfois, de ut dico ('comme je dis'). L'emploi de ces deux verbes, qui réfèrent de même à l'acte de dire, suggère bien qu'il s'agit là d'un phénomène énonciatif :

[15] Cic. Att. 1, 16, 8 (= exemple [6]) : 


\begin{tabular}{|c|c|c|c|c|c|c|}
\hline $\begin{array}{l}\text { Idem } \\
\text { le_même-NOM.SG }\end{array}$ & $\begin{array}{l}\text { ego } \\
\text { moi-NOM }\end{array}$ & $\begin{array}{l}\text { ille } \\
\text { DEM.NOM.SG.M }\end{array}$ & $\begin{array}{l}\text { (non } \\
\text { ne_pas }\end{array}$ & $\begin{array}{l}\text { enim } \\
\text { en_effet }\end{array}$ & $\begin{array}{l}\text { mihi } \\
\text { moi-DAT }\end{array}$ & $\begin{array}{l}\text { uide-or } \\
\text { sembler-PRS.1SG }\end{array}$ \\
\hline $\begin{array}{l}\text { insolenter } \\
\text { insolemment }\end{array}$ & $\begin{array}{l}\text { riari } \\
\text { vanter-INF }\end{array}$ & $\begin{array}{ll}\text { cum } & \text { de } \\
\text { quand } & \text { de }\end{array}$ & $\begin{array}{l}\text { me } \\
\text { moi-ABL }\end{array}$ & $\begin{array}{l}\text { apud } \\
\text { devant }\end{array}$ & $\begin{array}{l}\text { te } \\
\text { toi-ACC }\end{array}$ & \\
\hline
\end{tabular}

\begin{tabular}{|c|c|c|c|c|c|c|}
\hline $\begin{array}{l}\text { loqu-or, } \\
\text { parler-PRS.1SG }\end{array}$ & $\begin{array}{l}\text { in } \\
\text { dans }\end{array}$ & $\begin{array}{l}\text { ea } \\
\text { DEM.ABL.SG.F }\end{array}$ & $\begin{array}{l}\text { praesertim } \\
\text { surtout }\end{array}$ & $\begin{array}{l}\text { epistul-a } \\
\text { lettre-ABL.SG }\end{array}$ & & \\
\hline qu-am & nol-o & & ali-is & leg-i) & idem & inquam \\
\hline REL.ACC.SG.F & ne_pas & _vouloir-PRS.1SG & autre-DAT.PL & lire-INF.PASS & le_même-NOM.SG & $\overline{\text { dis-je }}$ \\
\hline
\end{tabular}

$\begin{array}{lllll}\text { ego } & \text { recre-au-i } & \text { adflict-os } & \text { anim-os } & \text { bon-orum } \\ \text { moi-NOM } & \text { ranimer-PFT.1SG } & \text { abattu-ACC.PL } & \text { esprit-ACC.PL } & \text { bon-GEN.PL }\end{array}$

$\begin{array}{lll}\begin{array}{l}\text { un-umqu-emque } \\ \text { chacun-ACC }\end{array} & \begin{array}{l}\text { confirm-ans, } \\ \text { rassurer-PART.PRS.NOM.SG }\end{array} & \text { excit-ans. } \\ \text { stimuler-PART.PRS.NOM.SG }\end{array}$

«Ce fut moi encore (car je n'ai pas le sentiment de manquer à la modestie quand je parle de moi devant toi, surtout dans une lettre que je ne veux pas qu'on lise à d'autres), ce fut moi, dis-je, qui rendis courage une fois encore aux bons citoyens, rassurant, stimulant chacun. » (L.-A. Constans)

33. En effet, si une reprise est nécessaire, c'est bien sur un plan énonciatif, pour que l'allocutaire, ou le lecteur, puisse suivre plus facilement le fil du discours. En employant le verbe dire ('dis-je', 'comme je le dis'), Cicéron reprend alors les termes de H1, à la fois en usage et en mention, et par là même, les souligne. C'est ainsi que la répétition sur la chaîne syntagmatique, qui paraît redondante sur un plan structurel, prend toute sa signification. C'est donc la linéarisation du discours, dans l'espace à l'écrit, dans le temps à l'oral, qui entraîne ce genre de phénomène, permettant indirectement de délimiter l'incidente sur son bord droit.

\subsubsection{Absence de terme spécifique}

Toutefois, la présence de tels marqueurs n'est pas systématique. En particulier, lorsque le premier fragment de la phrase hôte et l'incidente sont de faible extension, on n'observe aucun terme spécifique qui marquerait la reprise de la phrase hôte :

[16] Cic. Att. 1, 18, 2 :

$\begin{array}{lllllll}\text { Atque } & \text { hi } & \text { (nol-o } & \text { enim } & \text { te } & \text { permou-eri) } & \text { non } \\ \text { et } & \text { DEM.NOM.PL.M } & \text { ne_pas_vouloir-PRS.1SG } & \text { en_effet } & \text { toi-ACC } & \text { ébranler-INF.PASs } & \text { ne_pas }\end{array}$

sunt permolest-i.

être-PRS.3PL très_grave-NOM.PL

«D'ailleurs (car je ne voudrais pas que tu t'en effraies), ils [ses chagrins domestiques] ne sont pas très graves. » (L.-A. Constans)

34. Ainsi, dans cet exemple, après suppression de l'incidente, la phrase hôte reste parfaitement grammaticale, et ne présente pas de particularité due à la présence de l'incidente. On peut supposer que la taille de l'incidente, qui est assez courte, joue ici un 
rôle, en ce qui concerne le facteur de la mémoire chez l'interlocuteur ou le lecteur : sa longueur ne suffit pas à faire oublier le début de la phrase hôte.

35. Cependant, on peut remarquer qu'en l'absence de terme de reprise spécifique, n'importe quel terme n'occupe pas la première place de $\mathrm{H} 2^{6}$. Dans ces cas, en effet, il s'agit de termes qui permettent de délimiter indirectement l'incidente par rapport à la phrase hôte tels que négations ou verbes qui annoncent l'entrée dans une proposition différente.

\subsubsection{Anacoluthe}

On trouve aussi, cependant, le cas inverse : loin de n'avoir aucune influence syntaxique sur la phrase hôte, l'incidente, par sa présence, crée alors dans la phrase hôte des anomalies grammaticales que l'on appelle traditionnellement anacoluthes. Dans ces cas, l'incidente a souvent une extension plus grande, qui explique en partie la perturbation syntaxique.

36. Tout d'abord, il arrive que la syntaxe du deuxième segment de la phrase hôte soit induite non par son premier segment, mais par l'incidente elle-même :

[17] Cic. Tusc. 1, 40 :

$\begin{array}{lllll}\begin{array}{l}\text { Num } \\ \text { est-ce-que_par_hasard }\end{array} & \begin{array}{l}\text { igitur } \\ \text { donc }\end{array} & \begin{array}{l}\text { dubita-mus } \\ \text { douter-PRS.1PL }\end{array} & \begin{array}{l}\text { sicut } \\ \text { comme }\end{array} & \begin{array}{l}\text { pleraque } \\ \text { la_plupart_de-ACC.PL.N }\end{array} \quad \text { bien_que }\end{array}$

$\begin{array}{lllll}\text { hoc } & \text { quidem } & \text { minime } \\ \text { DEM.ACC.SG.N } & \begin{array}{l}\text { certes } \\ \text { très_peu }\end{array} & \begin{array}{l}\text { persuade-nt } \\ \text { prouver-PRS.3PL }\end{array} & \begin{array}{l}\text { enim } \\ \text { en_effet }\end{array} & \begin{array}{l}\text { mathematic-i-i- } \\ \text { mathématicien-NOM.PL }\end{array}\end{array}$

$\begin{array}{llllll}\text { terr-am } & \text { in } & \text { medi-o } & \text { mund-o } & \text { sitam } & \text { ad } \\ \text { terre-ACC.SG } & \text { dans } & \text { milieu-ABL.SG } & \text { monde-ABL.SG } & \text { placée-ACC.SG } & \text { par_rapport_à }\end{array}$

$\begin{array}{llllll}\text { uniuers-i } & \text { cael-i } & \text { complex-um } & \text { quasi } & \text { punct-i } & \text { instar } \\ \text { univers-GEN.SG } & \text { ciel-GEN.SG } & \text { enchaînement-ACC.SG } & \text { pour_ainsi_dire } & \text { point-GEN.SG } & \text { grandeur }\end{array}$

\begin{tabular}{|c|c|c|}
\hline obtinere, & quod & 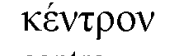 \\
\hline
\end{tabular}

« Pour entrer donc en matière, admettons d'abord un fait, qui pour nous-mêmes, quoique nous doutions presque de tout, n'est pas douteux, car les mathématiciens le prouvent : que la terre n'est, à l'égard de l'univers entier, que comme un point, qui, étant placé au milieu, en fait le centre. » (M. Nisard)

37. Ainsi, dans cet exemple, le deuxième segment de la phrase hôte semble se rattacher syntaxiquement à l'incidente. De fait, la proposition infinitive contenant obtinere ('occuper') paraît davantage dépendre de persuadent ('prouvent') que de dubitamus ('nous doutons'); en effet, alors que le verbe persuadere ('prouver') gouverne de manière générale une proposition infinitive, le verbe dubitare ('douter') entraîne normalement une subordonnée interrogative indirecte. On peut alors formuler deux hypothèses : soit on peut supposer que la proposition infinitive se rattache à dubitamus, construction rare influencée par la présence du verbe persuadere dans l'incidente; soit on peut considérer, de manière plus radicale, que la construction

\footnotetext{
${ }^{6} \mathrm{H} 2$ désigne la partie de la phrase hôte qui suit l'incidente.
} 
infinitive dépend directement de l'incidente, sous l'effet d'une rupture de construction ; car à ce moment-là, l'insertion de l'incidente se résout finalement en une intégration syntaxique, qui, sur un plan structurel, laisse le premier segment de la phrase hôte incomplet. Dans ce dernier cas, la structure générale de la phrase paraît alors bancale, dans la mesure où l'insertion, sur un autre plan, de l'incidente, au lieu de s'achever par une reprise de la phrase hôte, perd progressivement son statut d'insertion, étant donné que l'incidente devient finalement la phrase principale.

38. Toutefois, un deuxième type d'anacoluthe, où la structure de la phrase hôte est modifiée non pas directement par des termes de l'incidente mais par sa présence même, par laquelle la construction initiale semble oubliée, se retrouve plus fréquemment :

[18] Cic. Att. 7, 2, 8 :

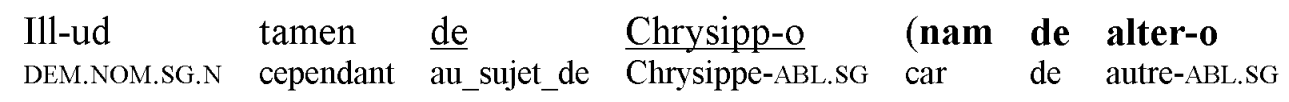

$\begin{array}{llllll}\text { ill-o } & \text { minus } & \text { sum admirat-us, } & \text { operari-o } & \text { hom-ine } ; & \text { sed } \\ \text { DEM.ABL.SG.M } & \text { moins } & \text { s'étonner-PFT.1sG } & \text { ouvrier-ABL.SG } & \text { homme-ABL.SG } & \text { mais }\end{array}$

$\begin{array}{llllll}\text { tamen } & \text { ne } & \text { ill-o } & \text { quidem } & \text { quicquam } & \text { improbius) } \\ \text { cependant } & \text { pas } & \text { DEM.ABL.SG.M } & \text { même } & \begin{array}{l}\text { quelque_chose-NOM.SG.N } \\ \text { plus_malhonnête-NOM.SG.N }\end{array}\end{array}$

$\begin{array}{llllll}\text { Chrysipp-um } & \text { uero } & \text { qu-em } & \text { ego } & \text { propter } & \text { litterul-arum } \\ \text { Chrysippe-ACC.SG } & \text { mais } & \text { REL.ACC.SG.M } & \text { moi-NOM } & \text { à_cause_de } & \text { petite_lettre-GEN.PL }\end{array}$

nesci-o quid libenter uid-i, in hon-ore habu-i

je_ne_sais REL.ACC.SG.N volontiers voir-PFT.1SG dans honneur-ABL.SG avoir-PFT.1sG

$\begin{array}{lllll}\text { disced-ere } & \text { a } & \text { puer-o } & \text { inscient-e } & \text { me ! } \\ \text { s'éloigner-INF } & \text { de } & \text { enfant-ABL.SG } & \text { ignorer-PART.PRS.ABL.SG } & \text { moi-ABL }\end{array}$

«Sur Chrysippe pourtant — de l'autre, je m'étonne moins, un tâcheron : bien que lui aussi dépasse les bornes de l'improbité-, mais que ce Chrysippe, qu'un vague frottis de littérature m'a fait gentiment accueillir, honorer, quitte mon fils à mon insu !» (Jean Bayet)

39. Ainsi, dans cet exemple, de Chrysippo ('au sujet de Chrysippe') est reformulé, après l'incidente, par l'accusatif Chrysippum ('Chrysippe'), ce qui crée un changement de construction de la phrase hôte au cours de sa linéarisation, si bien que le premier segment illud tamen de Chrysippo ('cela cependant au sujet de Chrysippe') n'est finalement plus intégré syntaxiquement à la phrase. Mais contrairement aux exemples précédents, du fait de la répétition du terme désignant Chrysippe, le deuxième segment de la phrase hôte est syntaxiquement autonome, si bien qu'il semble plutôt s'agir ici d'un phénomène d'interruption et de recommencement de la construction, plutôt que d'une modification progressive de celle-ci. Il est donc difficile de comprendre ce genre de phrase en analysant sa structure d'ensemble, mais il semble plus aisé de l'étudier sous l'angle de vue de sa genèse et de sa linéarisation. De fait, ce type de phénomène montre que la linéarisation de la phrase, dans l'espace ou dans le temps, peut avoir une grande influence sur sa construction globale, qui, au lieu d'être fixée, se modifie progressivement. 
40. Dans tous ces exemples, l'incidente n'est donc pas simplement insérée dans la phrase hôte, mais crée des modifications syntaxiques de divers ordres dans la phrase hôte, ce qui entraîne une certaine dépendance, inhabituelle, de celle-ci par rapport à l'incidente, à tel point que, dans certains cas, la distinction d'une phrase hôte et d'une incidente s'avère difficile à déterminer clairement. Les frontières deviennent floues et la structure d'ensemble bancale. En termes moins métaphoriques, et sous un angle de vue temporel, la construction syntaxique initiale de la phrase hôte est modifiée par l'apparition de l'incidente, ce qui crée une structure d'ensemble peu cohérente.

41. Mais il existe encore un autre type d'anomalie créée par la présence de l'incidente, que l'on pourrait qualifier de pléonasme :

[19] Cic. Verr. 4, 29 :

$\begin{array}{lllllll}\text { In } & \text { Sicili-a } & \text { quidem } & \text { cum } & \text { ess-em, } & \text { sic } & \text { a } \\ \text { dans } & \text { Sicile-ABL.SG } & \text { certes } & \text { alors_que } & \text { être-SUBJ.IMPFT.1sG } & \text { ainsi } & \text { de }\end{array}$

$\begin{array}{lllll}\begin{array}{l}\text { Centuripin-is, } \\ \text { habitant_de_Centorbi-ABL.PL }\end{array} & \text { sic } & \text { ainsi } & \text { ceter-is } & \text { audi-ebam } \\ \text { tous_les_autres-ABL.PL } & \text { entendre_dire-IMPFT.1sG }\end{array}$

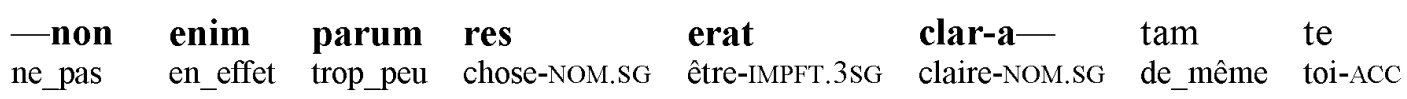

$\begin{array}{lllll}\text { h-as } & \text { phaler-as } & \text { a } & \text { Phylarch-o } & \text { Centuripin-o } \\ \text { DEM.ACC.PL.F } & \text { phalère-ACC.PL } & \text { à } & \text { Phylarque-ABL.SG } & \text { de_Centorbi-ABL.SG }\end{array}$

$\begin{array}{llllllll}\text { abstul-isse } & \text { dic-ebant } & \text { quam } & \text { ali-as } & \text { item } & \text { nobil-is } & \text { ab } & \text { Arist-o } \\ \text { enlever-INF.PFT } & \text { dire-IMPFT.3PL } & \text { comme } & \text { autre-ACC.PL } & \text { de_même } & \text { précieux-ACC.PL } & \text { à } & \text { Ariste-ABL.SG }\end{array}$

Panhormitan-o, qu-am terti-as a Cratipp-o Tyndaritan-o. de_Palerme-ABL.SG comme troisième-ACC.PL à Cratippe-ABL.SG de.Tyndaris-ABL.SG

«Pendant mon séjour en Sicile, j'ai ouï dire aux habitants de Centorbi et à tous les Siciliens (car la chose n'était rien moins qu'un mystère), que tu les as enlevées à Phylarque, comme tu en as pris d'autres non moins précieuses à Ariste de Palerme et d'autres encore à Cratippe de Tyndaris. » (P. C. B. Gueroult)

42. Ainsi, dans cet exemple, si l'on considère la phrase hôte sans tenir compte de l'incidente, on constate qu'elle comprend deux verbes principaux dont dépend une seule proposition infinitive : la proposition tam te has phaleras a Phylarcho Centuripino abstulisse quam alias item nobilis ab Aristo Panhormitano, quam tertias a Cratippo Tyndaritano ('que tu les as enlevées à Phylarque, comme tu en as pris d'autres non moins précieuses à Ariste de Palerme et d'autres encore à Cratippe de Tyndaris') semble gouvernée aussi bien par le verbe audiebam ('j'ai entendu dire') que par le verbe dicebant ('on disait'). Les deux verbes, non coordonnés et de même signification, semblent, de manière redondante, jouer le même rôle syntaxique. Il ne s'agit pas exactement ici d'anacoluthe, dans la mesure où la construction syntaxique reste grammaticale, étant donné que les deux verbes commandent la même construction, mais la double saturation de la même fonction est bien une situation anomale créé par la présence de l'incidente. Il s'agit là encore d'un phénomène dû à la linéarisation de la phrase: le locuteur éprouve le besoin, afin de faciliter la compréhension de son 
interlocuteur ou de son lecteur, d'exprimer à nouveau le verbe principal, étant donné que la présence de l'incidente crée une distance entre la proposition infinitive et le premier verbe principal.

43. Avec les phénomènes d'anacoluthe, on semble donc parvenir aux limites de la délimitation de l'incidente : en effet, les perturbations syntaxiques que celle-ci entraîne rendent souvent difficile la détermination d'une frontière droite, voire la distinction même d'une incidente et d'une phrase hôte.

44. De tels cas, toutefois, ne sont pas si fréquents, et de surcroît, la frontière est ici ténue entre incidence et disfluence, qui est courante dans le discours oral. Ces cas ne sont donc pas surprenants dans une rhétorique de l'imitation de l'oral à l'écrit et paraissent marginaux par rapport au cas prototypique de l'incidence.

45. Dans les occurrences prototypiques d'incidentes, il est au contraire possible de délimiter précisément les frontières de l'incidente, que les marqueurs de frontière examinés plus haut soient présents, ou que d'autres phénomènes signalent indirectement le début ou la fin de l'incidente sur la chaîne syntagmatique. A partir de là, il est légitime de s'interroger sur les positions que peuvent occuper les incidentes dans la phrase hôte : peut-on repérer une certaine régularité et prédictibilité dans les sites occupés par l'incidente?

\section{Place de l'incidente dans la phrase hôte}

\subsection{Sa position dans la chaîne linéaire de la phrase}

Eduard Schwyzer ${ }^{7}$ parle de Prosthothese, de Mesothese et d'Opisthothese, selon que la parenthèse se situe au début, au milieu, ou à la fin de la phrase. Il se place donc à l'échelle de la phrase pour distinguer les sites périphériques des sites incis ${ }^{8}$ des incidentes. Toutefois, si, comme on l'a affirmé, l'insertion est l'un des critères d'identification spécifiques de l'incidente, il est difficile de prendre en compte les places initiale et finale de la phrase, qui ne peuvent être propres à l'insertion. Dès lors, il faut ou bien remettre en question le caractère obligatoire du critère de l'insertion, ou bien rejeter l'existence des prosthothèses et les opisthothèses, ou encore réexaminer la notion de phrase. En effet, il n'est pas toujours évident de décider des frontières de la phrase, surtout en latin, où elles ne sont pas marquées par la ponctuation, et où l'intonation n'est pas contrôlable. On peut saisir les difficultés que cela implique dans l'exemple suivant :

[20] Cic. Comm. Pet. 33 :

$\begin{array}{llll}\text { Primum } & \text { cognosc-e } & \text { equit-es } & \text { (pauc-i } \\ \text { tout_d'abord } & \text { faire_la_connaissance-IMP.2SG } & \text { cavalier-ACC.PL } & \text { peu_nombreux-NOM.PL }\end{array}$

enim sunt), deinde appet-e (multo enim facilius en_effet être-PRS.3PL ensuite attirer-IMP.2SG beaucoup en_effet plus_facilement

$\begin{array}{llllll}\text { illa } & \text { adulescentul-orum } & \text { ad } & \text { amiciti-am } & \text { aetas } & \text { adiung-itur). } \\ \text { DEM.NOM.SG.F } & \text { jeunes_gens-GEN.PL } & \text { à } & \begin{array}{l}\text { amitié-ACC.SG } \\ \text { âge-NOM.SG }\end{array} & \text { rallier-PASS.PRS.3SG }\end{array}$

\footnotetext{
${ }^{7}$ Schwyzer : 1939, p. 33.

${ }^{8}$ Il s'agit là de la terminologie de Marandin : 1999.
} 
«En premier lieu, fais la connaissance des cavaliers (ils sont peu nombreux), puis fais leur conquête (ces très jeunes gens sont à un âge où on se laisse beaucoup plus facilement gagner à l'amitié). » (L.-A. Constans)

46. De fait, à première vue, on pourrait ou bien considérer qu'il s'agit d'une seule phrase composée de deux propositions coordonnées par les connecteurs primum ('tout d'abord') et deinde ('ensuite') —à ce moment-là, la première parenthèse serait bien insérée-, ou bien estimer qu'il s'agit de deux phrases distinctes introduites par des connecteurs dans ce cas, les deux parenthèses se trouveraient en fin de phrase, et leur statut d'incidente serait problématique, dans la mesure où elles ne répondent pas au critère de l'insertion. Mais on peut alors se rendre compte qu'il ne s'agit plus véritablement d'un problème syntaxique, mais plutôt d'un problème discursif. En effet, c'est au niveau discursif que l'on peut argumenter pour savoir si ces deux propositions forment un seul ou deux énoncés, grâce à des critères prosodiques —non disponibles, cependant, en latin-, sémantiques et pragmatiques. On perçoit ainsi que, si l'on ne se place plus au niveau de la phrase syntaxique, mais au niveau de l'énoncé, les prosthothèses et les opisthothèses deviennent peut-être compatibles avec le critère de l'insertion.

47. Cependant, si l'on suit Jean-Marie Marandin', il est également possible de parler d'inclusion de l'incident dans l'hôte lorsqu'il se place en tête ou en queue de l'hôte, si l'on raisonne avec une définition particulière de l'hôte: «l'hôte est envisagé structuralement et sa structure implique une idée de frontière qui ne se confond pas avec le premier ou le dernier terminal ${ }^{10}$ de l'hôte ». Ainsi, un adverbe placé en tête de phrase peut être indépendant syntaxiquement par rapport à l'hôte tout en étant inclus dans sa structure. Cependant dans le cas d'une proposition - cas auquel nous nous limitons ici -, il parait bien plus difficile de savoir si, en position initiale ou finale, elle est incluse ou non dans la structure de l'hôte. Sans critère supplémentaire, il paraît tout aussi justifié de supposer qu'il s'agit de proposition juxtaposée que de proposition insérée; il semble même que l'on soit de nouveau confronté au même problème que précédemment : ce sont à nouveau des critères discursifs qui pourraient apporter des éléments de précision.

48. Mais cette analyse de Jean-Marie Marandin suggère au moins qu'il est difficile, pour étudier la position de l'incidente dans la phrase hôte, de raisonner en termes de placement parmi les terminaux de la chaîne linéaire, sans référer au fait qu'il s'agit d'une structure composée de syntagmes. Par exemple, après un rapide examen de plusieurs exemples d'incidentes, on pourrait supposer que l'incidente se place très souvent en deuxième position de la chaîne de la phrase hôte :

[21] Cic. Att. 5, 20, 3 :

\footnotetext{
${ }^{9}$ Marandin : 1999, p. 6.

${ }^{10}$ Le terme «terminal » réfère aux nœuds terminaux d'un arbre syntaxique. Un terminal correspond donc à chaque mot d'une phrase envisagée comme séquence linéaire. J.-M. Marandin suggère ici que l'hôte n'est pas assimilé à la chaîne de terminaux qui le réalisent, mais envisagé structurellement.
} 


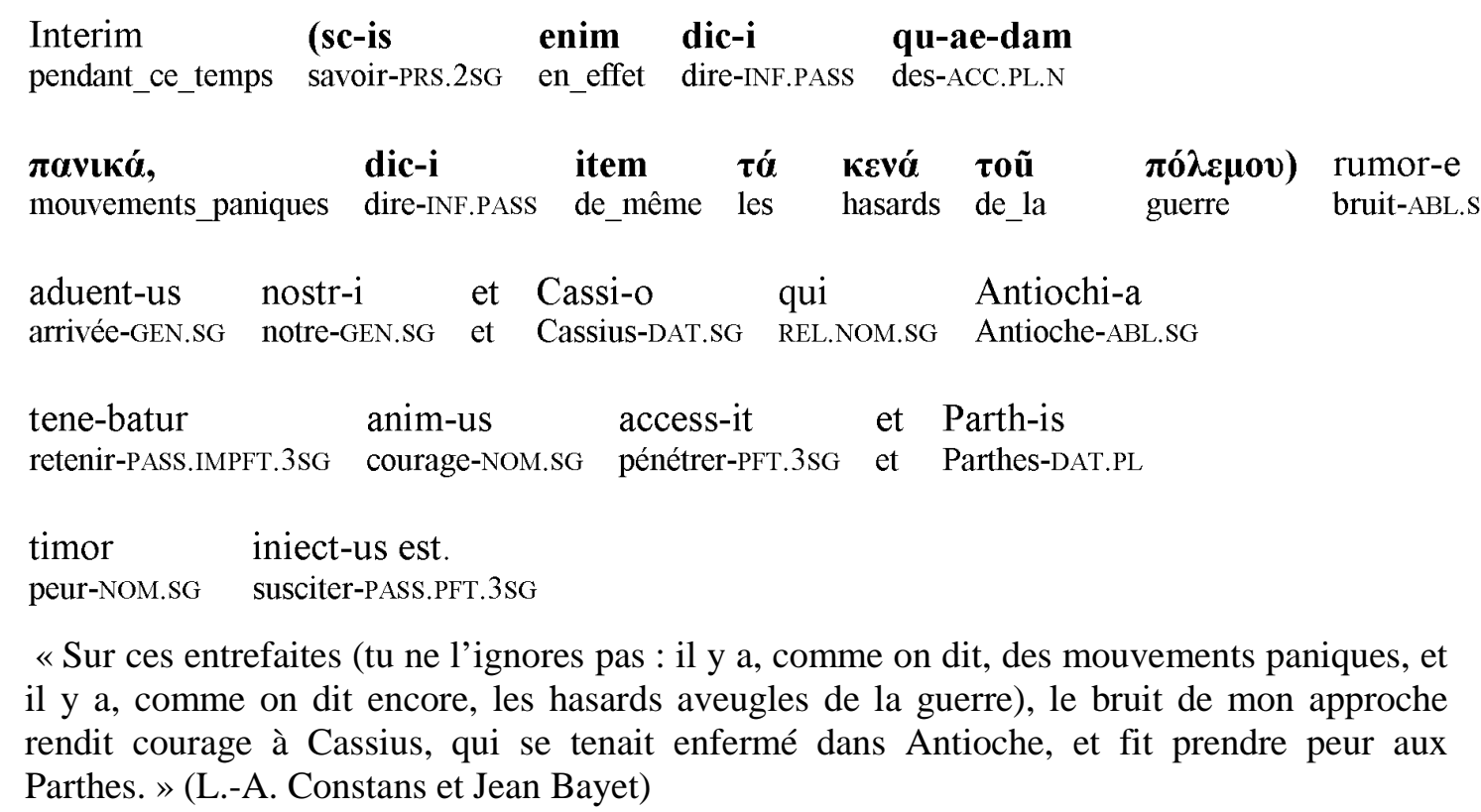

«Sur ces entrefaites (tu ne l'ignores pas : il y a, comme on dit, des mouvements paniques, et il y a, comme on dit encore, les hasards aveugles de la guerre), le bruit de mon approche rendit courage à Cassius, qui se tenait enfermé dans Antioche, et fit prendre peur aux Parthes. » (L.-A. Constans et Jean Bayet)

49. Toutefois, l'exemple suivant suggère plutôt que l'incidente peut se placer après le premier syntagme de la phrase hôte, et non après son premier mot :

[22] Cic. Att. 5, 21, 7 :

\begin{tabular}{|c|c|c|c|}
\hline $\begin{array}{l}\text { Qu-a } \\
\text { REL.ABL.SG.F }\end{array}$ & $\begin{array}{l}\text { insul-a } \\
\text { île-ABL.SG }\end{array}$ & 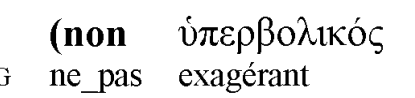 & $\begin{array}{ll}\text { sed } & \text { uerissime } \\
\text { mais de_manière_tout_à_fait_véridique }\end{array}$ \\
\hline $\begin{array}{l}\text { loqu-or) } \\
\text { parler-PRS.1sG }\end{array}$ & $\begin{array}{l}\text { numm-us } \\
\text { sou-NOM.SG }\end{array}$ & $\begin{array}{l}\text { null-us } \\
\text { aucun-NOM.SG }\end{array}$ & $\begin{array}{l}\text { eroga-bitur. } \\
\text { demander-PASS.FUT.3SG }\end{array}$ \\
\hline
\end{tabular}

50. De fait, qua ex insula ('à cette île') correspond à un syntagme nominal complément du verbe erogabitur ('sera demandé'), et constitue, en terme de position dans la chaîne, le premier syntagme nominal de la phrase. Pour s'interroger avec pertinence sur la position de l'incidente dans la phrase hôte, on peut donc supposer qu'il faut envisager la phrase hôte comme une structure composée de groupes fonctionnels.

\subsection{Sa position dans la structure de la phrase hôte}

La question est donc de savoir à quels endroits de la phrase hôte, par rapport aux syntagmes qui la constituent, peut se placer l'incidente. Dans la plupart des études consacrées aux parenthèses dans diverses langues, il est affirmé qu'il n'existe pas de contraintes en ce qui concerne leur position ${ }^{11}$. Pourtant, comme le suggère Jean-Marie Marandin ${ }^{12}$, il est aisé de montrer, du moins dans les langues vivantes ${ }^{13}$, que l'incidence

\footnotetext{
${ }^{11}$ Voir par exemple, à propos du français, Pétillon-Boucheron : 2002, p. 204 : «il n’y a pas, à proprement parler d'impossibilités ou d'interdits quant aux positions du décroché : toutes les positions sont disponibles pour l'opération de décrochement (typo)graphique ».

${ }^{12}$ Voir Marandin : 1999, p. 3.

${ }^{13}$ Le problème en latin est, bien sûr, qu'on ne dispose pas de locuteurs qui pourraient établir un jugement d'agrammaticalité ou de grammaticalité.
} 
peut être l'objet de jugements de malformation, ce qui implique qu'il existe des contraintes de bonne formation, et que le placement de l'incidente n'est peut-être pas aussi aléatoire qu'il paraît.

51. Ainsi, apparemment, c'est principalement au niveau des frontières de groupes de la phrase hôte que l'incidente peut se placer. C'est donc cette intuition qu'il faut essayer d'examiner avec plus de détails.

\subsubsection{A l'échelle de la phrase}

\section{a) Entre deux propositions \\ $\alpha$ - Coordonnées}

On peut distinguer des groupes fonctionnels à plusieurs niveaux dans la phrase hôte. Ceux de plus haut niveau correspondent aux propositions coordonnées dans la phrase hôte. Or on observe un grand nombre d'incidentes placées entre deux propositions coordonnées, juste avant la conjonction de coordination précédant la deuxième proposition :

[23] Cic. Fam. 13, 62 (à P. Silius) :

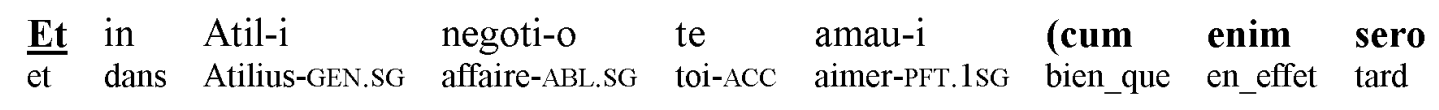

$\begin{array}{lllll}\begin{array}{l}\text { uen-issem, } \\ \text { venir-SUBJ.PQPFT.1SG }\end{array} & \begin{array}{l}\text { tamen } \\ \text { cependant }\end{array} & \begin{array}{l}\text { honest-um } \\ \text { distingué-ACC.SG }\end{array} & \begin{array}{l}\text { equ-item } \\ \text { chevalier-ACC.SG }\end{array} & \begin{array}{l}\text { benefici-o } \\ \text { service-ABL.SG }\end{array}\end{array}$

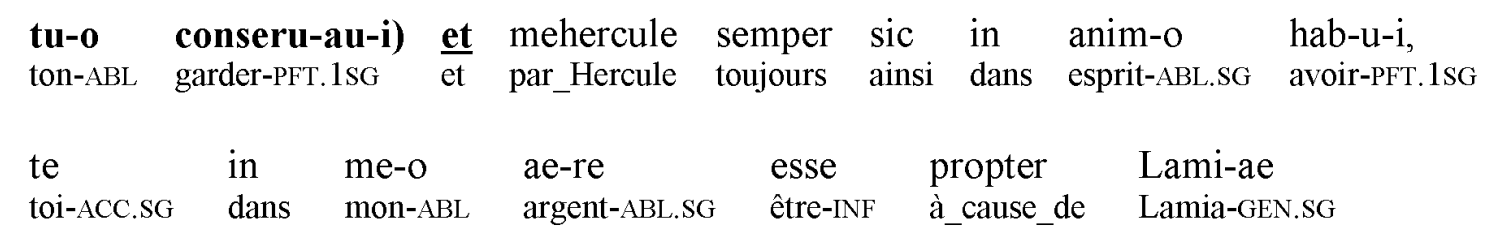

nostri coniunction-em et singular-em necessitudin-em.

notre-GEN.SG amitié-ACC.SG et particulier-ACC.SG lien-ACC.SG

«Si l'affaire d'Atilius t'a rendu cher à mon cœur (venu trop tard, c'est grâce à toi que cependant j'ai sauvé ce chevalier distingué), j'ai aussi, pardieu, toujours été persuadé que tu étais tout à moi, à cause de l'amitié et des liens tout particuliers qui nous attachent à notre cher Lamia. » (L.-A. Constans et Jean Bayet)

\section{$\beta$ - Subordonnées}

En outre, la place située entre deux propositions subordonnées est, de même, souvent occupée par des incidentes, ce qui suggère que la frontière entre deux propositions, quelle que soit la force du lien syntaxique qui les unit, est propice à l'insertion d'incidentes. De fait, tous les types de subordonnées sont ici concernés, qu'un corrélatif soit présent ou non, et quelle que soit la fonction occupée par la subordonnée dans la phrase hôte. De plus, l'incidente se place indifféremment en fin de subordonnée ou en fin de principale.

[24] Cic. Att. 6, 5, 3 : 


$\begin{array}{llllll}\text { Etsi } & \text { exercit-um } & \text { infirm-um } & \text { habe-bam } & \text { auxili-a } & \text { sane } \\ \text { bien_que } & \text { armée-ACC.SG } & \text { faible-ACC.SG } & \text { avoir-IMPFT.1SG } & \text { auxiliaire-ACC.PL } & \text { tout_à_fait }\end{array}$

\begin{tabular}{|c|c|c|c|c|}
\hline $\begin{array}{l}\text { bon-a, } \\
\text { bon-ACC.PL }\end{array}$ & $\begin{array}{l}\text { sed } \\
\text { mais }\end{array}$ & $\begin{array}{l}\text { ea } \\
\text { DEM.ACC.PL.N }\end{array}$ & $\begin{array}{l}\text { Galat-arum, } \\
\text { Galate-GEN.PL }\end{array}$ & $\begin{array}{l}\text { Pisid-arum, } \\
\text { Pisidien-GEN.PL }\end{array}$ \\
\hline
\end{tabular}

$\begin{array}{lllllll}\begin{array}{l}\text { (h-aec } \\ \text { DEM.NOM.PL.N }\end{array} & \begin{array}{l}\text { enim } \\ \text { en_effet }\end{array} & \begin{array}{l}\text { sunt } \\ \text { ETRE-PRS.3PL }\end{array} & \begin{array}{l}\text { nostr-a } \\ \text { nos-NOM.PL.N }\end{array} & \begin{array}{l}\text { rob-ora), } \\ \text { force-NOM.PL }\end{array} & \begin{array}{l}\text { tamen } \\ \text { cependant }\end{array} & \begin{array}{l}\text { esse } \\ \text { être-INF }\end{array} \\ \begin{array}{lllll}\text { offici-um } \\ \text { devoir-ACC.SG }\end{array} & \begin{array}{l}\text { me-um } \\ \text { mon-ACC }\end{array} & \begin{array}{l}\text { put-aui } \\ \text { penser-PFT.1SG }\end{array} & \begin{array}{l}\text { exercit-Um } \\ \text { armée-ACC.SG }\end{array} & \begin{array}{l}\text { hab-ere } \\ \text { tenir-INF }\end{array} & \begin{array}{l}\text { quam proxime } \\ \text { le_plus_près_possible }\end{array}\end{array}$

host-em.

ennemi-ACC.SG

«Bien que je ne dispose que d'une armée débile, avec, il est vrai, de bons auxiliaires, mais Galates, Pisidiens, Lyciens — car ce sont là mes troupes d'élite-, j'ai cru de mon devoir de tenir mon armée aussi près que possible de l'ennemi.. » (L.-A. Constans)

[25] Cic. Q. Fr. 1, 3, 8 (= exemple [1]) :

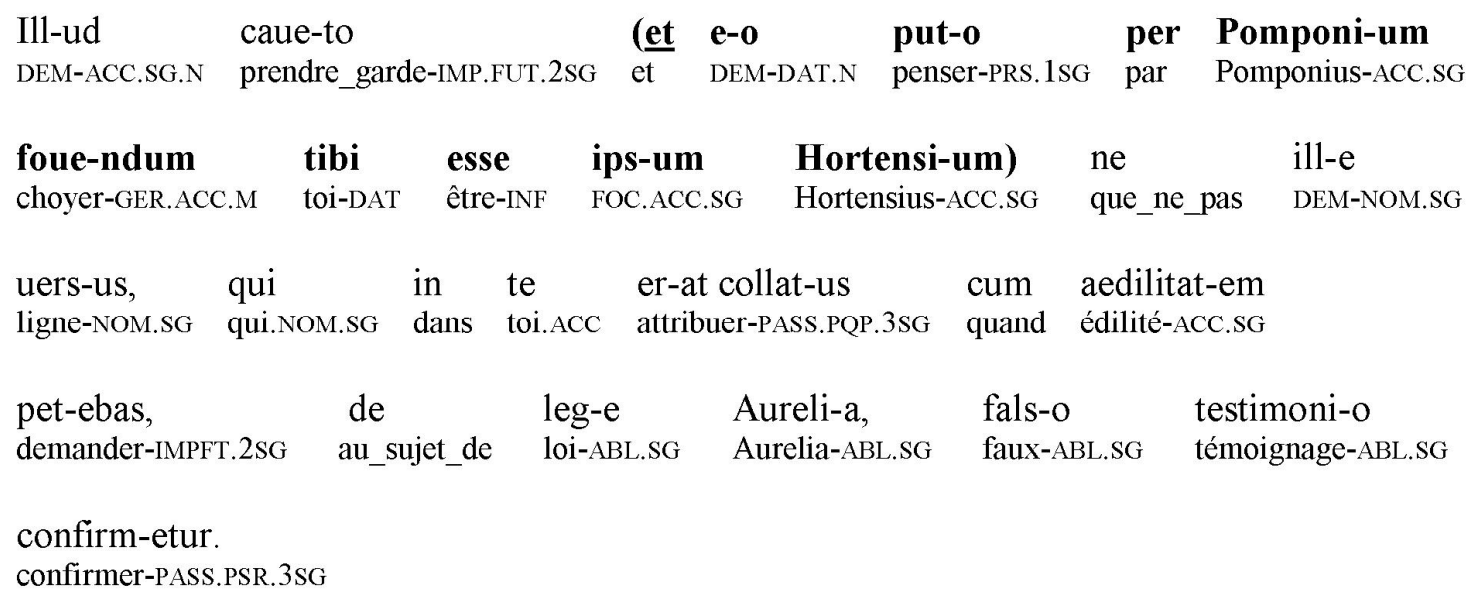

«Il te faut aussi prendre garde (et pour cela tu devras, je crois, par l'intermédiaire de Pomponius, cultiver Hortensius lui-même) qu'un faux témoignage ne vienne confirmer que tu es l'auteur de cette épigramme sur la loi Aurelia qui t'avait été attribuée quand tu briguais l'édilité. » (L.-A. Constans)

52. Les incidentes s'insèrent donc de manière récurrente à la frontière de deux propositions, qu'elles soient coordonnées ou subordonnées. Ce phénomène peut s'expliquer par le fait que les deux propositions sont syntaxiquement reliées de manière moins étroite autrement dit à un niveau hiérarchique supérieur- que ne le sont les éléments qui constituent chaque proposition; puisque chaque proposition forme un ensemble cohérent, il semble plus attendu que l'incidente se place entre ces deux ensembles, sans désolidariser ses éléments, qu'à l'intérieur de ces ensembles. Ainsi, il s'agit peut-être de l'endroit de la chaine où les éléments peuvent le plus facilement être mis à distance, d'une part parce que chacun des éléments, placé à un niveau hiérarchique élevé, forme un tout à lui seul — bien que non autonome dans le cas de la subordination-, et d'autre 
part, parce que le lien qui unit les deux ensembles est assez marqué — par un terme spécifique - pour que la mise à distance ne nuise pas à la bonne formation de la phrase.

53. Toutefois, si l'on peut regrouper ces deux cas au niveau formel, il faut les distinguer au niveau fonctionnel : tandis que les propositions coordonnées n'ont pas de fonction syntaxique l'une par rapport à l'autre, ce n'est pas le cas des propositions subordonnées, dans la mesure où une proposition subordonnée possède une fonction grammaticale dans la proposition dite principale : elle peut ainsi entrer dans le paradigme d'un circonstant, dans le cas, par exemple, des subordonnées conditionnelles ou temporelles, ou d'un actant du verbe principal, dans le cas des subordonnées complétives ou interrogatives indirectes. Du point de vue fonctionnel, il ressort donc que les incidentes se positionnent de manière privilégiée entre les constituants principaux de la phrase hôte. Cela nous conduit alors à nous demander si les incidentes se placent de la même manière à la frontière des différents syntagmes nominaux fonctionnels.

\section{b) Entre deux syntagmes nominaux}

Les incidentes peuvent se placer à la frontières de différents groupes fonctionnels : entre deux compléments circonstanciels, entre le sujet et l'attribut du sujet, entre le complément et le verbe, entre le verbe et le sujet.

[26] Cic. Q. fr. 3, 1, 1 :

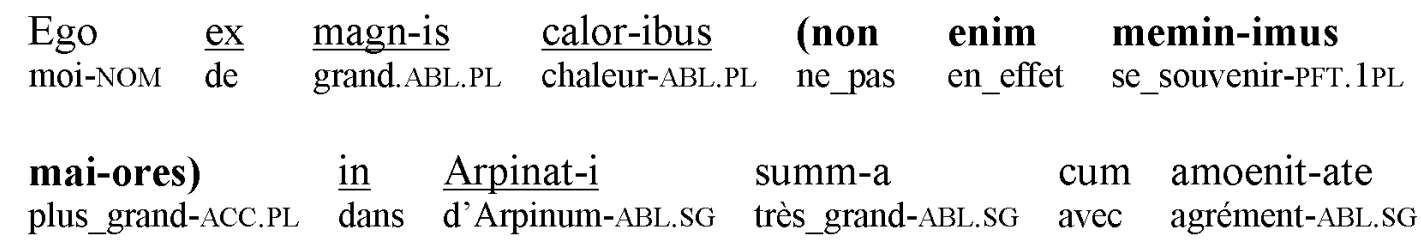

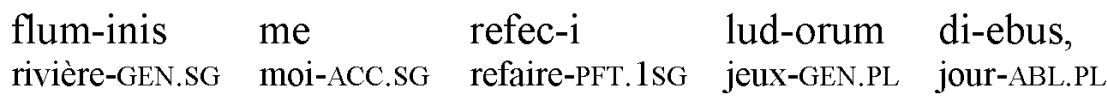

Philotim-o tribuli-bus commendat-is.

Philotime-DAT.SG de_la_même_tribu-ABL.PL confié-ABL.PL

«J'ai réparé les fatigues des grandes chaleurs (nous n'avons pas souvenir d'en avoir vu de pires) dans ma villa d'Arpinum, en jouissant des agréments de la rivière, pendant le temps des jeux, après avoir confié les gens de ma tribu à Philotime. » (L.-A. Constans)

54. Il apparaît ainsi que les incidentes semblent pouvoir se placer entre tous les constituants immédiats de la phrase hôte. Un site d'insertion paraît donc potentiellement présent entre les différents grands groupes fonctionnels de la phrase hôte, qui, de fait, possèdent une unité, marquée souvent de manière morphologique par le marquage casuel.

\subsubsection{A l'échelle du constituant}

\section{a) Entre deux syntagmes nominaux}

Pourtant, on observe également que plusieurs incidentes se placent au milieu de groupes fonctionnels, créant une certaine discontinuité dans ces groupes. De telles positions pourraient alors donner l'impression, au premier abord, d'un placement syntaxiquement aléatoire des incidentes : 
[27] Cic. Att. 1, 18, 6 :

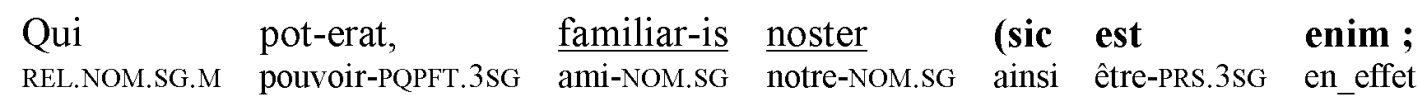

$\begin{array}{llllll}\text { uol-o } & \text { te } & \text { hoc } & \text { scire) } & \text { Pompei-Us } & \text { togul-am } \\ \text { vouloir-PRS.1SG } & \text { toi-ACC } & \text { DEM.ACC.SG.N } & \text { savoir-INF } & \text { Pompée-NOM.SG } & \text { petite_toge-ACC.SG }\end{array}$

$\begin{array}{lllll}\text { ill-am } & \text { pict-am } & \text { silenti-o } & \text { tue-tur } & \text { su-am. } \\ \text { DEM.ACC.SG.F } & \text { brodée-ACC.SG } & \text { silence-ABL.SG } & \text { garder-PRs.3SG } & \text { sa-ACC.SG }\end{array}$

«Quelqu'un aurait pu l'être [un véritable homme d'Etat], mon ami Pompée (car il est mon ami, et je veux que tu le saches); mais il se contente de protéger, en gardant un prudent silence, sa belle petite toge brodée. » (L.-A. Constans)

55. De fait, l'incidente désolidarise ici, par son insertion, les éléments du groupe sujet, en mettant à distance familiaris noster ('mon ami') et Pompeius ('Pompée'). On peut ainsi avoir l'impression que le placement de ce type d'incidentes répond à des contraintes d'ordre non pas syntaxique, mais exclusivement sémantique : la parenthèse correspond à un commentaire sur l'emploi de l'adjectif qui précède.

56. Cependant, si on se place à l'échelle du syntagme nominal, on constate que les éléments séparés par l'insertion de l'incidente sont aussi, à un niveau hiérarchique inférieur, des groupes fonctionnels. En effet, Pompeius correspond à une expansion du syntagme nominal qui est, le plus souvent, appelée apposition. Ainsi, ce type d'incidentes se positionne aussi à la frontière de deux groupes, si l'on adopte non plus le point de vue de la phrase entière, mais celui du groupe fonctionnel. On peut donc faire l'hypothèse que le placement de l'incidente répond aussi à des exigences syntaxiques, d'après lesquelles les sites d'insertion se trouvent aux frontières des groupes, aux différents niveaux hiérarchiques de la phrase.

57. Au niveau du groupe fonctionnel, on observe, de même qu'au niveau de la phrase, de nombreuses occurrences d'incidentes situées entre deux éléments coordonnés, qu'un coordonnant soit exprimé ou non :

[28] Cic. Att. 5, 21, 9 (= exemple [10]) :

$\begin{array}{llllll}\text { Id-ibus } & \text { Februari-is, } & \text { qu-o } & \text { di-e } & \text { h-as } & \text { litter-as } \\ \text { ide-ABL.PL } & \text { de_février-ABL.PL. } & \text { REL.ABL.SG } & \text { jour-ABL.SG } & \text { DEM.ACC.PL. } & \text { lettre-ACC.PL }\end{array}$

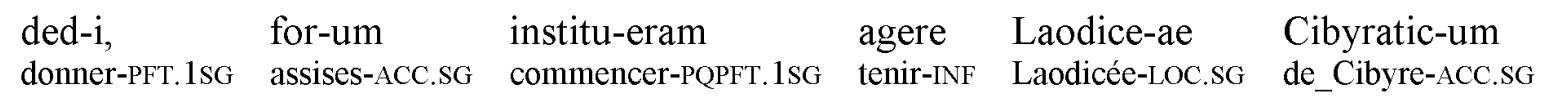

et Apamens-e; ex Id-ibus Marti-is ibidem

et d'Apamée-ACC.SG à_partir_de ide-ABL.PL de_mars-ABL.PL au_même_endroit

Synnadens-e, Pamphyli-um (tum Phemi-o dispici-am

de_Synnade-ACC.SG de_Pamphylie-ACC.SG alors Phemius-DAT.SG examiner-FUT.1SG

$\begin{array}{lll}\boldsymbol{\kappa} \boldsymbol{\varepsilon} \boldsymbol{\rho} \boldsymbol{\alpha} \mathbf{\alpha}) & \text { Lycaoni-um, } \\ \text { flûte } & \begin{array}{l}\text { Isauric-um. } \\ \text { de_Lycaonie-ACC.SG }\end{array} & \text { d'Isaurie-ACC.SG }\end{array}$ 
«Aujourd'hui 13 février, je commence à tenir mes assises à Laodicée pour les gens de Cibyra et d'Apamée ; à partir du 15 mars, toujours à Laodicée, je jugerai pour Synnade, la Pamphylie (c'est alors que j'aurai l'œil à une flûte pour Phémius), la Lycaonie, l'Isaurie. » (L.-A. Constans et Jean Bayet)

58. On retrouve le cas observé plus haut, à une échelle plus petite : au lieu que les incidentes se placent entre deux propositions de niveau hiérarchique supérieur, elles se placent, ici, entre deux éléments coordonnés à l'intérieur d'un groupe fonctionnel. Il n'en reste pas moins qu'elles se situent également à une frontière, même si cette frontière n'est pas au même niveau syntaxique.

59. En outre, comme précédemment à l'échelle de la phrase, les éléments séparés par l'incidente peuvent également, à l'échelle du groupe fonctionnel, correspondre aussi bien à des syntagmes nominaux qu'à des propositions. Dans le cas intermédiaire où l'incidente se place entre une relative et son antécédent, elle se trouve ainsi entre un syntagme nominal et une proposition. Dans l'exemple suivant, elle sépare, à l'intérieur du complément du nom peruersitas ('l'extravagance'), le nom noyau hominum ('des hommes') de son expansion, qui prend la forme d'une relative :

[29] Cic. Fam. 1, 7, 7 :

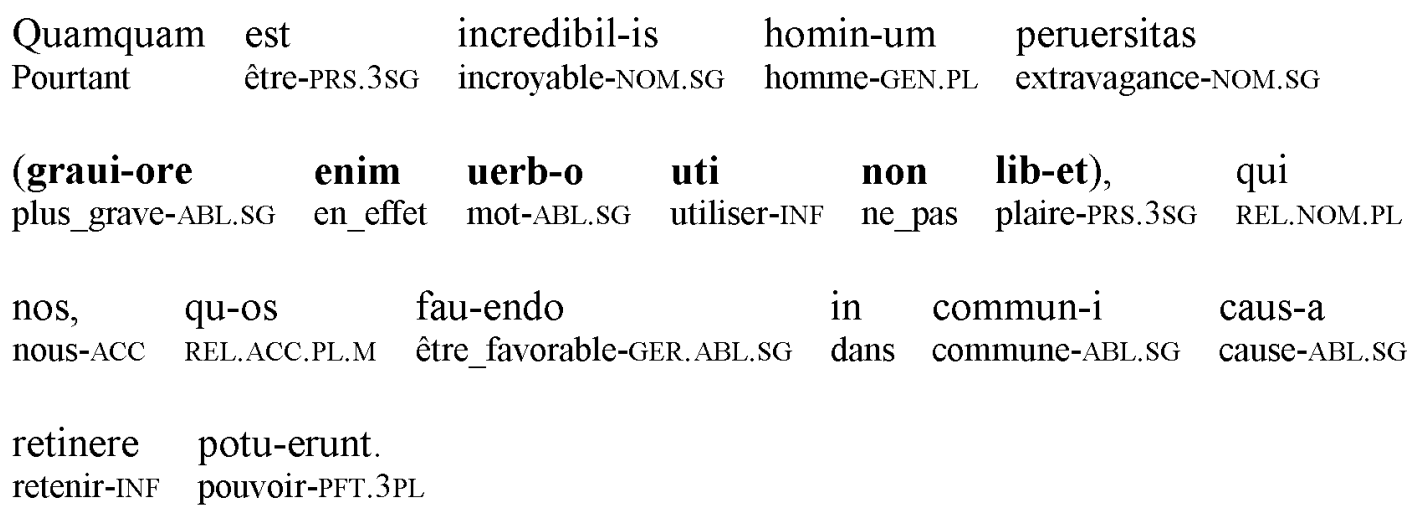

«Pourtant, il y a des gens dont le manque de bon sens (je ne veux pas employer d'expression plus forte) est incroyable : notre cause était commune et ils auraient pu, en montrant de bons sentiments, me retenir. » (L.-A. Constans)

\section{b) Entre deux propositions}

De manière similaire, on constate que des incidentes peuvent se placer entre deux propositions subordonnées situées à l'intérieur d'un groupe fonctionnel :

[30] Cic. Q. fr. 3, 2, 2 :

\begin{tabular}{|c|c|c|c|c|c|}
\hline $\begin{array}{l}\text { Tene-o } \\
\text { tenir-PRS.1SG }\end{array}$ & $\frac{\text { uel }}{\text { ou_bien }}$ & $\frac{\text { quod }}{\text { parce_que }}$ & $\begin{array}{l}\text { nol-o } \\
\text { ne_pas_vouloir-P }\end{array}$ & $\begin{array}{l}\text { cum } \\
\text { contre }\end{array}$ & $\begin{array}{l}\text { Pompei-o } \\
\text { Pompée-ABL.sc }\end{array}$ \\
\hline $\begin{array}{l}\text { pugnare } \\
\text { combattre-INF }\end{array}$ & $\begin{array}{l}\text { (satis } \\
\text { assez }\end{array}$ & $\begin{array}{l}\text { est } \\
\text { être-PRS.3sG }\end{array}$ & $\begin{array}{l}\text { qu-od } \\
\text { REL.NOM.SG.N }\end{array}$ & $\begin{array}{l}\text { inst-at } \\
\text { menacer-PRS.3SG }\end{array}$ & $\begin{array}{l}\text { de } \\
\text { au_sujet_de }\end{array}$ \\
\hline $\begin{array}{l}\text { Milo-ne) } \\
\text { Milon-ABL.SG }\end{array}$ & $\frac{\text { uel }}{\text { ou_bien }}$ & $\frac{\text { quod }}{\text { parce_que }}$ & $\begin{array}{l}\text { iudic-es } \\
\text { juge-ACC.PL }\end{array}$ & $\begin{array}{l}\text { uull-os } \\
\text { qucun-ACC.PL }\end{array}$ & $\begin{array}{l}\text { be-mus. } \\
\text { oir-PRs.1PL }\end{array}$ \\
\hline
\end{tabular}


« Je me retiens [de me charger de l'accusation] : je ne veux pas entrer en lutte avec Pompée (c'est assez de ce qui menace au sujet de Milon), et puis, nous n'avons pas de juges. » (L.-A. Constans)

60. Ainsi, les incidentes, malgré les apparences, obéissent à certaines contraintes syntaxiques quant à leur placement. En effet, elles se placent toujours à la frontière des groupes fonctionnels, qu'ils prennent la forme de syntagmes nominaux ou de propositions. Certes, dans la mesure où il peut s'agir de groupes fonctionnels aussi bien au niveau de la phrase qu'à celui des différents constituants, les sites d'insertion d'incidentes se révèlent être nombreux, ce qui explique cette apparente absence de contraintes syntaxiques quant au placement. Toutefois, après un examen plus attentif, on remarque que toutes les positions ne sont pas représentées ${ }^{14}$, ce qui nous pousse à formuler l'hypothèse selon laquelle les incidentes se positionnent à la frontière des différents groupes fonctionnels.

61. En outre, il serait intéressant de préciser cette hypothèse, en se demandant s'il existe des critères permettant d'expliquer la distribution entre les différents sites d'insertion.

\subsection{Distribution et portée}

En effet, si l'on se restreignait exclusivement à l'hypothèse précédemment formulée, en supposant que la seule contrainte de placement d'une incidente est de se situer entre deux groupes fonctionnels de la phrase hôte, cela laisserait une large part au hasard, étant donné le nombre de frontières de groupes qu'il existe dans une phrase hôte. La question est donc de savoir s'il existe des contraintes distributionnelles parmi les différents sites d'insertion mis au jour.

\subsubsection{La notion de domaine}

Pour y répondre, la notion de domaine, proposée par Jean-Marie Marandin ${ }^{15}$, nous semble être tout à fait adéquate. Elle permet, en effet, de saisir les contraintes sémanticosyntaxiques, liées au phénomène de portée, qui régissent le placement des incidentes.

62. Tout d'abord, il ressort de nos exemples que l'incidente peut porter, au niveau sémantico-syntaxique, sur des éléments d'ampleurs différentes : ainsi, la portée de l'incidente peut correspondre à la phrase hôte dans son ensemble, à un groupe de mots, ou à un seul terme. Plus précisément, en termes syntaxiques, l'incidente paraît pouvoir porter sur l'ensemble de la phrase hôte ou sur l'un de ses constituants ou sousconstituants. C'est cette portée que l'on peut alors désigner par le terme de domaine syntaxique de l'incidente.

63. A partir de là, la première règle que l'on peut formuler est la suivante : l'incidente se place toujours à l'intérieur ou à la périphérie de son domaine syntaxique. Dès lors, on peut différencier les incidentes portant sur la phrase hôte dans son ensemble, qui se situent à la frontière de l'un de ses groupes fonctionnels, et celles portant sur un constituant, qui se placent à l'intérieur ou à la périphérie de ce constituant.

\footnotetext{
${ }^{14}$ En latin, cela ne restera toutefois qu'une hypothèse, dans la mesure où le corpus des textes est limité et où l'on ne peut pas procéder à des tests de grammaticalité chez des locuteurs natifs.

${ }^{15}$ Voir Marandin : 1999, p. 15.
} 
64. Ainsi, l'incidente se place toujours à l'intérieur ou à la périphérie du groupe fonctionnel sur lequel elle porte :

[31] Cic. Att. 1, 18, 6 (= exemple [27]) :

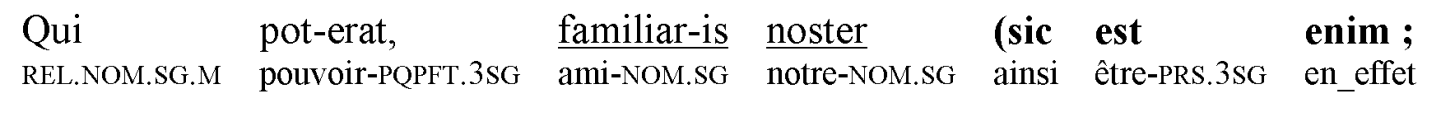

$\begin{array}{llllll}\text { uol-o } & \text { te } & \text { hoc } & \text { scire) } & \text { Pompei-us } & \text { togul-am } \\ \text { vouloir-PRS.1SG } & \text { toi-ACC } & \text { DEM.ACC.SG.N } & \text { savoir-INF } & \text { Pompée-NOM.SG } & \text { petite_toge-ACC.SG }\end{array}$

$\begin{array}{lllll}\text { ill-am } & \text { pict-am } & \text { silenti-o } & \text { tue-tur } & \text { su-am. } \\ \text { DEM.ACC.SG.F } & \text { brodée-ACC.SG } & \text { silence-ABL.SG } & \text { garder-PRS.3SG } & \text { sa-ACC.SG }\end{array}$

«Quelqu'un aurait pu l'être [un véritable homme d'Etat], mon ami Pompée (car il est mon ami, et je veux que tu le saches); mais il se contente de protéger, en gardant un prudent silence, sa belle petite toge brodée. » (L.-A. Constans)

65. Par exemple, ici, l'incidente, qui porte sur un élément du groupe sujet, se place donc dans ce groupe sujet. Située plus à droite, donc hors de son domaine, elle ne pourrait plus porter sur familiaris noster ('mon ami').

66. On peut cependant noter que les termes en relation endophorique avec l'incidente n'appartiennent pas obligatoirement à son domaine syntaxique :

[32] Cic. Att. 6, 9, 2:

$\begin{array}{llllll}\text { Hanc, } & \text { qu-ae } & \text { mehercule } & \text { mihi } & \text { magn-o } & \text { dolor-i } \\ \text { DEM.ACC.SG.F } & \text { REL.NOM.SG.F } & \text { par_Hercule } & \text { moi-DAT } & \text { grand-DAT.SG } & \text { douleur-DAT.SG }\end{array}$

$\begin{array}{lllll}\text { est } & \text { (dilex-i } & \text { enim } & \text { homin-em), } & \text { procur-a, } \\ \text { être-PRS.3SG } & \text { aimer-PFT.1SG } & \text { en_effet } & \text { homme-ACC.SG } & \text { prendre_soin-IMP.2SG }\end{array}$

quantul-a-cumque est, Precian-am hereditat-em prorsus

si_petit_que-NOM.SG.F être-PRS.3sg de_Precius-ACC.SG héritage-ACC.SG tout_à_fait

$\begin{array}{lll}\text { ill-e } & \text { ne } & \text { atting-at. } \\ \text { DEM.NOM.SG.M } & \text { que_ne_pas } & \text { toucher-SUBJ.PRS.3SG }\end{array}$

«[...] Ce que m'a laissé Précius (j'en ai grand deuil, par Hercule : je l'aimais), prends-en soin, si faible que soit l'héritage, que notre homme n'aille pas y toucher du bout des doigts. ${ }^{16} \gg$ (Jean Bayet)

67. En effet, dans cet exemple, l'incidente se situe bien à la périphérie de son domaine syntaxique, sur son bord droit : de fait, elle porte sur la relative quae me hercule mihi magno dolori est ('qui me fait beaucoup souffrir, par Hercule'). Cependant, le référent de hominem ('l'homme') est à déduire de la relation cataphorique qu'entretient hominem avec Precianam ('de Precius'), adjectif qui n'appartient pas au domaine syntaxique que nous venons d'identifier. La notion de domaine syntaxique est donc bien à comprendre au sens étroit d'élément sur lequel porte l'incidente.

\footnotetext{
${ }^{16}$ Notons que le parenthésage de la traduction de Jean Bayet et celui de l'édition latine sont différents : le traducteur choisit de mettre 'j'en ai grand deuil, par Hercule' entre parenthèses alors que ce fragment de phrase ne figure pas entre parenthèses dans l'édition du texte latin.
} 


\subsubsection{La place dans le domaine}

Cette première règle posée, on peut s'interroger sur la place que peut prendre l'incidente dans son domaine ; mais cela s'avère bien plus difficile à déterminer. Nous ne pouvons que dégager certaines tendances, d'autant plus que le nombre d'exemples examinés reste trop faible pour établir des règles sûres.

\section{a) Incidentes à un constituant}

Dans le cas des incidentes à un groupe fonctionnel, on observe que l'incidente se place généralement sur le bord droit du groupe ou du sous-groupe sur lequel elle porte.

[33] Cic. Att. 3, 13, 2 :

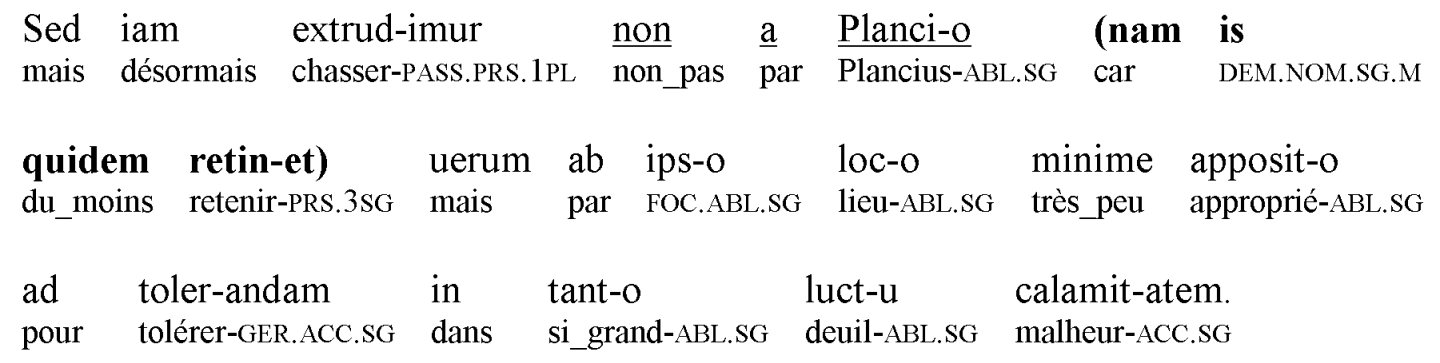

«Mais à présent j'en suis chassé, non par Plancius (car lui, il me retient), mais par les inconvénients du séjour même, qui est aussi peu fait que possible pour rendre le malheur tolérable à un homme aussi affligé que moi. » (L.-A. Constans)

68. Ainsi, l'incidente porte sur le sous-groupe non a Plancio ('non par Plancius') et se place sur son bord droit.

69. Un cas particulier, toutefois, pourrait théoriquement mettre en doute cette tendance : c'est celui des incidentes métalinguistiques. En effet, lorsqu'une incidente porte sur un terme pris en mention, elle se place en général immédiatement après ce terme, sans que soit prise en compte la distinction en groupes fonctionnels ${ }^{17}$. Les exemples d'incidentes métalinguistiques montrent, de fait, que l'incidente se positionne à la droite immédiate du terme sur lequel elle porte :

[34] Cic. Fam. 1, 7, 7 (= exemple [29]) :

\footnotetext{
${ }^{17}$ Pour ce qui concerne le français, Sabine Pétillon-Boucheron constate ainsi que «le contenu du X-décroché est lié à la position qu'il occupe dans la phrase et plus cette position semble inhospitalière, plus l'on a de chance d'y trouver un X-décroché de nature métalinguistique ». Voir Pétillon-Boucheron : 2002, pp. 231-232.
} 


\begin{tabular}{|c|c|c|c|c|}
\hline Quamquam & incredibil-is & in-um & peruersitas & \\
\hline être-PRS.3SG & incroyable-NOM.SG & ne-GEN.PL & extravagance & NOM.SG \\
\hline $\begin{array}{l}\text { (graui-ore } \\
\text { plus_grave-ABL.SG }\end{array}$ & $\begin{array}{l}\text { uerb-o } \\
\text { mot-ABL.SG }\end{array}$ & $\begin{array}{l}\text { non } \\
\text { ne_pas }\end{array}$ & $\begin{array}{l}\text { lib-et), } \\
\text { plaire-PRS.3sG }\end{array}$ & $\begin{array}{l}\text { qui } \\
\text { REL.NOM.PL }\end{array}$ \\
\hline $\begin{array}{l}\text { qU-OS } \\
\text { REL.ACC.PL.M }\end{array}$ & $\begin{array}{l}\text { au-endo } \\
\text { tre_favorable-GER.ABL.SG }\end{array}$ & dans & $\begin{array}{l}\text { mmun-i } \\
\text { nmune-ABL.SG }\end{array}$ & $\begin{array}{l}\text { caus-a } \\
\text { cause-ABL.SG }\end{array}$ \\
\hline $\begin{array}{l}\text { potu-erunt. } \\
\text { pouvoir-PFT.3PL }\end{array}$ & & & & \\
\hline
\end{tabular}

«Pourtant, il y a des gens dont le manque de bon sens (je ne veux pas employer d'expression plus forte) est incroyable : notre cause était commune et ils auraient pu, en montrant de bons sentiments, me retenir. » (L.-A. Constans)

70. Il est toutefois difficile, dans les exemples attestés, de vérifier l'hypothèse proposée. De fait, dans tous les cas que nous avons rencontrés, les incidentes qui portent sur des termes en mention se placent toujours immédiatement après ces termes, mais ce placement correspond en même temps à la frontière droite d'un groupe fonctionnel. A défaut d'autres exemples plus discriminants, cela ne reste donc qu'une hypothèse théorique, qui ne peut donc pas infirmer la tendance mise au jour, selon laquelle l'incidente à un syntagme se place à la frontière droite de ce syntagme.

\section{b) Incidentes à la phrase entière}

Si l'on étendait cette tendance aux cas où l'incidente porte sur la phrase hôte dans son ensemble, il faudrait en conclure que l'incidente peut se placer en fin de phrase. Cela nous ouvre deux pistes : d'une part, cela pourrait nous inciter à réexaminer les cas d'opisthothèses, que nous avons tout d'abord rejetés, dans la mesure où ils ne répondaient pas au critère de l'insertion. D'autre part, et inversement, cela suggère que les règles de placement — s'il en existe bien qui soient régulières - ne sont peutêtre pas identiques dans le cas de l'incidence à un constituant et dans le cas de l'incidence à toute une phrase. Ces deux pistes semblent toutefois s'exclure - soit la règle de placement sur la frontière droite est valable à tous les niveaux, et à ce moment-là, il existe des opisthothèses, soit les règles de position sont différentes à l'échelle du constituant et à celle de la phrase, et alors, on peut toujours rejeter les opisthothèses. Cependant, un moyen terme semble également possible : les règles sont plus complexes à l'échelle de la phrase, mais incluent la règle précédente, ce qui impliquerait que les opisthothèses existent; il nous faut donc examiner en détail ces hypothèses.

\section{$\alpha$ - La deuxième position}

Tout d'abord, plusieurs exemples nous permettent au moins de constater qu'une autre position, très fréquente, se rencontre lorsque l'incidente porte sur toute la phrase hôte : il s'agit de la deuxième position :

[35] Cic. Fam. 7, 2 (à Marius), 3 : 


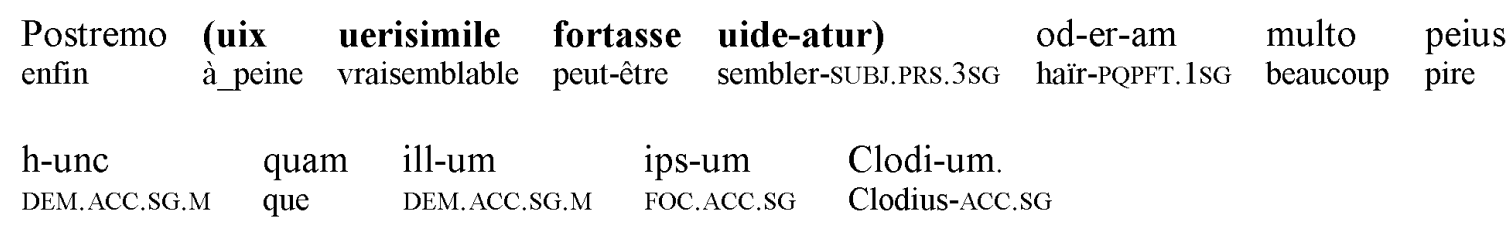

«Enfin (il se peut que la chose paraisse difficilement vraisemblable), je hais bien plus cet homme [Bursa] que je ne haïssais Clodius lui-même. » (L.-A. Constans)

71. Ainsi, dans cet exemple, l'incidente porte manifestement sur l'ensemble de la phrase hôte ; il serait de toute façon très difficile de considérer qu'elle porte uniquement sur le premier terme. Or dans les deux cas, elle se place en deuxième position, juste après un connecteur : la portée de l'incidente la suit donc, contrairement à ce que nous avons pu observer dans les cas d'incidence à un groupe fonctionnel.

72. Il faut cependant essayer de préciser la notion de deuxième position. Tout d'abord, l'incidente ne suit pas forcément un connecteur, mais tout autre terme est également possible en première position. En outre, le placement de plusieurs particules en deuxième position, pour des raisons accentuelles notamment, est prioritaire sur le placement de l'incidente : la contrainte phonologique l'emporte sur la contrainte syntaxique. Enfin, la notion de position est ici à comprendre en termes fonctionnels : il n'est pas question de mots, mais de constituants :

[36] Cic. Fam. 1, 9 (à Lentulus), 14 :

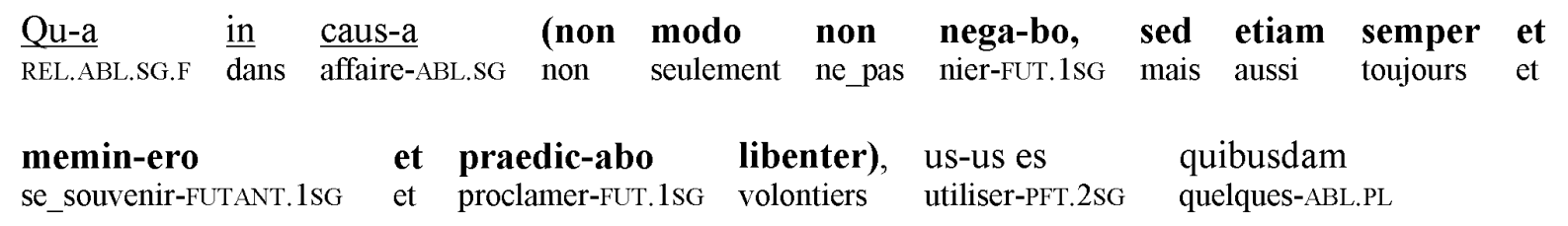

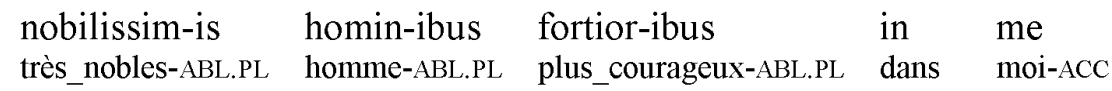

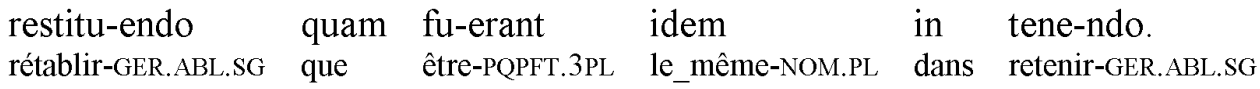

«Dans cette affaire (bien loin de le nier, toujours je m'en souviendrai et serai heureux de le proclamer), tu as trouvé le concours de certains hauts personnages qui se montrèrent plus courageux pour me rétablir qu'ils ne l'avaient été pour me maintenir. » (L.-A. Constans)

73. De fait, dans cet exemple, c'est dans la mesure où elle suit le complément circonstanciel qua in causa ('dans cette affaire') que l'incidente est placée en deuxième position : il s'agit de la deuxième position syntaxique.

74. Les règles de placement de l'incidente à l'échelle de la proposition indépendante ne semblent donc pas être identiques à celles qui prévalent à l'échelle du constituant : tandis que la position prototypique dans le premier cas paraît être la deuxième position, dans le deuxième cas, il s'agit de la frontière droite du domaine.

\section{$\beta$ - La dernière position}

Avant d'exclure la seconde hypothèse, qui conduirait à rejeter les opisthothèses, il nous faut cependant examiner certains exemples plus en détail : 
[37] Cic. Comm. Pet. 33 (= exemple [20]) :

$\begin{array}{llll}\text { Primum } & \text { cognosc-e } & \text { equit-es } & \text { (pauc-i } \\ \text { tout_d'abord } & \text { faire_la_connaissance-IMP.2SG } & \text { cavalier-ACC.PL } & \text { peu_nombreux-NOM.PL }\end{array}$

enim sunt), deinde appet-e (multo enim facilius

en_effet être-PRs.3PL ensuite attirer-IMP.2sG beaucoup en_effet plus_facilement

$\begin{array}{llllll}\text { illa } & \text { adulescentul-orum } & \text { ad } & \text { amiciti-am } & \text { aetas } & \text { adiung-itur). } \\ \text { DEM.NOM.SG.F } & \text { jeunes_gens-GEN.PL } & \text { à } & \text { amitié-ACC.SG } & \text { âge-NOM.SG } & \text { rallier-PASS.PRS.3SG }\end{array}$

«En premier lieu, fais la connaissance des cavaliers (ils sont peu nombreux), puis fais leur conquête (ces très jeunes gens sont à un âge où on se laisse beaucoup plus facilement gagner à l'amitié). » (L.-A. Constans)

75. En effet, on pourrait ici considérer que l'on a affaire à une opisthothèse, dans la mesure où la proposition multo enim facilius illa adulescentulorum ad amicitiam aetas adiungitur ('ces très jeunes gens sont à un âge où on se laisse beaucoup plus facilement gagner à l'amitié') semble correspondre à une incidente portant sur la phrase hôte deinde appete ('puis fais leur conquête'), à la fin de laquelle elle se trouve. On peut cependant considérer qu'il s'agit là de quatre phrases,

[Primum cognosce equites] [Pauci enim sunt] [Deinde appete][Multo enim facilius illa adulescentulorum ad amicitiam aetas adiungitur]

76. et non de deux,

[Primum cognosce equites (pauci enim sunt)] [Deinde appete (multo enim facilius illa adulescentulorum ad amicitiam aetas adiungitur)]

77. ou d'une seule :

[Primum cognosce equites (pauci enim sunt); deinde appete (multo enim facilius illa adulescentulorum ad amicitiam aetas adiungitur)].

78. De fait, si l'on considère qu'il s'agit de quatre phrases, il n'y a plus lieu de parler d'incidente placée en fin de phrase, c'est-à-dire à la droite du domaine que constitue l'hôte. Il s'agit donc d'établir les critères qui permettraient de déterminer le nombre de phrases mises en jeu ici : la notion de phrase pose en effet problème dans de tels cas. De fait, on ne peut pas ici observer d'insertion claire de l'incidente : il semble tout autant justifié de supposer une inclusion dans la structure de l'hôte $[\mathrm{H}(\mathrm{i})]^{18}$ qu'une juxtaposition de deux propositions $[\mathrm{H}][\mathrm{i}]$; de fait, dans la mesure où i correspond à une proposition, le cas reste ambigu du point de vue de la chaîne syntagmatique. Des critères énonciatifs deviennent alors déterminants: ainsi, la structure primum/deinde ('tout d'abord'/'ensuite') nous inciterait à considérer qu'il s'agit d'un seul énoncé, composé de deux propositions juxtaposées et de deux incidentes : [H1(i1); H2(i2)]. Mais sur un plan strictement syntaxique, cela reste ambigu, et les différentes structures proposées restent valables.

79. Un autre exemple de ce type permet toutefois d'apporter des éléments supplémentaires : [38] Cic. Q. Fr. 3, 4, 1 :

\footnotetext{
${ }^{18} \mathrm{H}$ symbolise la phrase hôte, et i l'incidente.
} 


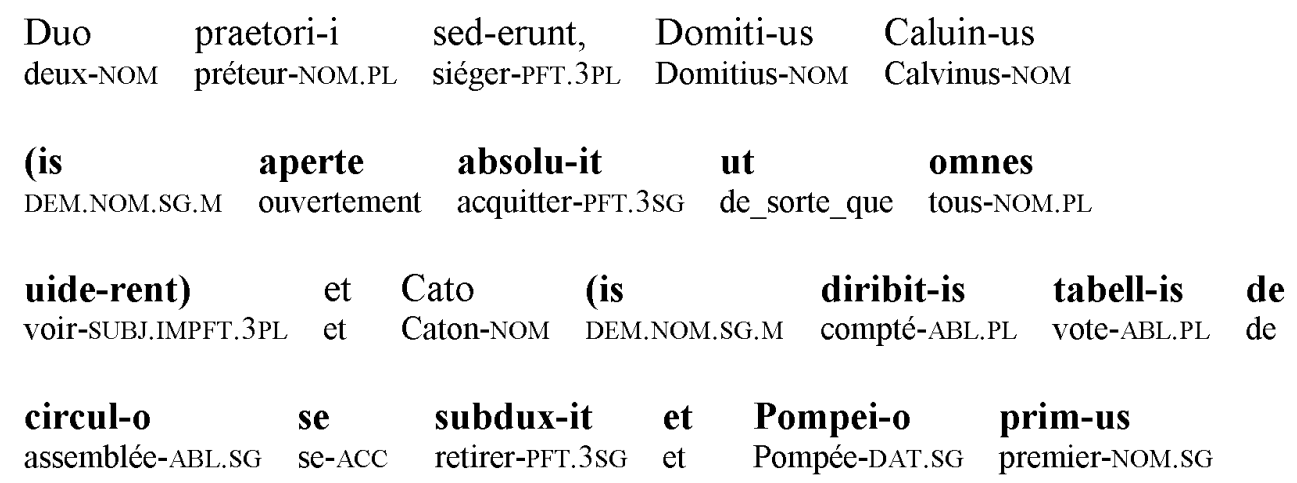

\section{nunti-auit).}

annoncer-PFT.3SG

«Il y avait deux anciens préteurs parmi eux, Domitius Caluinus et Caton : le premier a voté non coupable ostensiblement, de façon à ce que tout le monde le vît ; le second, dès qu' on eût dénombré les votes, s'est éclipsé pour aller annoncer à Pompée, le premier, la nouvelle. » (L.A. Constans)

80. De fait, on peut ici aussi observer, apparemment, une incidente placée en fin de la phrase hôte : is diribitis tabellis de circulo se subduxit et Pompeio primus nuntiauit ('lui, dès qu'on eût dénombré les votes, s'est éclipsé pour aller annoncer à Pompée, le premier, la nouvelle'). Ce cas, pourtant, est moins ambigu. En effet, cette incidente peut être mise en parallèle avec l'incidente précédente is aperte absoluit ut omnes uiderent ('lui a voté non coupable ostensiblement, de façon à ce que tout le monde le vît.'). Or, celle-ci se trouve manifestement à l'intérieur de la phrase hôte, dans la mesure où et Cato ('et Caton') ne peut constituer à lui seul une phrase. Dès lors, pour des raisons de symétrie structurelle, il est bien plus logique d'établir la structure suivante :

[Duo praetorii sederunt, Domitius Caluinus (is aperte absoluit ut omnes uiderent) et Cato (is diribitis tabellis de circulo se subduxit et Pompeio primus nuntiauit)],

81. plutôt que celle-ci :

[Duo praetorii sederunt, Domitius Caluinus (is aperte absoluit ut omnes uiderent) et Cato] [is diribitis tabellis de circulo se subduxit et Pompeio primus nuntiauit].

82. Dès lors, dans cette phrase, il est nettement préférable de considérer que nous avons affaire à une incidente placée en fin de la phrase hôte, ce qui implique l'existence des opisthothèses.

83. Cela ne signifie toutefois pas encore que les incidentes portant sur la phrase hôte dans son ensemble ne se placent pas seulement en deuxième position, mais également, comme à l'échelle des constituants, sur le bord droit du domaine de portée. En effet, dans l'exemple précédent, il est clair que les deux incidentes portent sur un constituant, respectivement Domitius Caluinus et Cato, et non sur la phrase hôte dans son ensemble.

84. Cependant, l'exemple suivant peut peut-être apporter un argument à cette dernière hypothèse :

[39] Cic. Att. 4, 9, 1 : 


$\begin{array}{llllll}\text { Sane } & \text { uel-im } & \text { scire } & \text { num } & \text { cens-um } & \text { impedi-ant } \\ \text { tout_à_fait } & \text { vouloir-SUBJ.PRS.1SG } & \text { savoir-INF } & \text { si } & \text { cens-ACC.SG } & \text { empêcher-SUBJ.PRS.3PL }\end{array}$

$\begin{array}{llllll}\text { tribun-i } & \text { di-ebus } & \text { uitiand-is } & \text { (est } & \text { enim } & \text { hic } \\ \text { tribun-NOM.PL } & \text { jour-ABL.PL } & \text { interdire-GER.ABL.PL } & \text { être-PRS.3SG } & \text { en_effet } & \text { DEM.NOM.SG.M }\end{array}$

$\begin{array}{llllll}\text { rumor) } & \text { tot-a } & \text {-que } & \text { de } & \text { censur-a } & \text { quid } \\ \text { bruit-NOM.SG } & \text { toute-ABL.SG } & \text { et } & \text { au_sujet_de } & \text { censure-ABL.SG } & \text { REL.ACC.SG.N }\end{array}$
ag-ant,
quid
cogit-ent.
faire-SUBJ.PRS.3PL REL.ACC.SG.N penser-SUBJ.PRS.3PL

«Je voudrais bien savoir si les tribuns mettent obstacle au cens en rendant tous les jours impropres à l'opération (c'est le bruit qui court ici), et ce qu'ils font, ce qu'ils méditent pour l'ensemble de la censure. » (L.-A. Constans)

85. En effet, l'incidente est ici, manifestement, insérée à l'intérieur de la phrase hôte, ce qui ne rend pas son statut d'incidente problématique :

[Sane uelim scire num censum impediant tribuni diebus uitiandis (est enim hic rumor) totaque de censura quid agant, quid cogitent].

86. Pourtant, la phrase resterait tout à fait grammaticale si l'on supprimait le dernier membre de phrase :

[Sane uelim scire num censum impediant tribuni diebus uitiandis (est enim hic rumor)].

87. Dans ce cas, il n'y aurait aucune raison de changer le statut de l'incidente. Dès lors, celle-ci se trouverait placée en fin de la phrase hôte: il s'agirait donc d'un cas d'opisthothèse.

88. Certes, ce n'est pas encore là un argument fort pour établir que des incidentes portant sur la phrase hôte peuvent occuper cette dernière position. En effet, ici, l'incidente porte bien plutôt sur la proposition interrogative indirecte num censum impediant tribuni diebus uitiandis ('si les tribuns mettent obstacle au cens en rendant tous les jours impropres à l'opération') qui constitue un groupe fonctionnel; c'est donc un domaine à la droite duquel l'incidente se place de manière régulière. Toutefois, le domaine de l'incidente correspond cette fois à une proposition, ce qui suggère peut-être que le cas est similaire quand il s'agit de la proposition que constitue la phrase hôte dans son ensemble. Sur un plan syntaxique, il est donc hautement probable que des incidentes portant sur la phrase hôte dans son ensemble occupent le bord droit du domaine, c'est-à-dire la fin de la phrase, même s'il faut sans doute recourir en dernière instance aux critères énonciatif et pragmatique pour opter entre les deux interprétations syntaxiques possibles.

89. En tout cas, s'il est très probable qu'une incidente portant sur l'ensemble de la phrase hôte puisse se placer sur son bord droit, cela signifie que les règles de placement des incidentes à l'échelle de la phrase hôte semblent plus complexes qu'à l'échelle du constituant. En effet, une incidente de ce type paraît pouvoir se placer aussi bien en deuxième position qu'en dernière position de la phrase hôte, tandis que tous les exemples concernant l'incidence à un groupe montraient un placement sur le bord droit du domaine. 
Toutefois, il serait plus prudent, avant de tirer cette conclusion, d'examiner une autre hypothèse, selon laquelle les règles de placement à l'échelle du constituant seraient également plus complexes que ce que nous avons pu établir jusque là. En effet, le cas présenté par l'exemple suivant, bien que rare, semble le suggérer :

[40] Cic. Att. 5, 12, 2 :

$\begin{array}{llllllll}\text { De } & \text { Messall-a } & \text { ad } & \text { te } & \text { statim } & \text { ut } & \text { aud-iu-i } & \text { de } \\ \text { au_sujet_de } & \text { Messala-ABL.sG } & \text { à } & \text { toi-ACC } & \text { aussitôt } & \text { quand } & \text { apprendre-PFT.1sG } & \text { au_sujet_de }\end{array}$

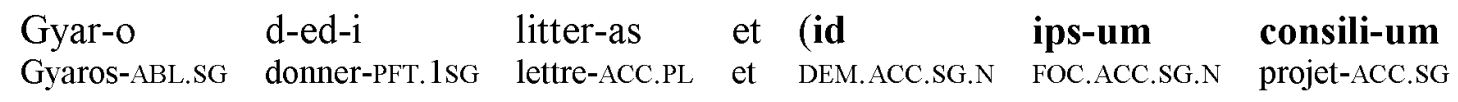

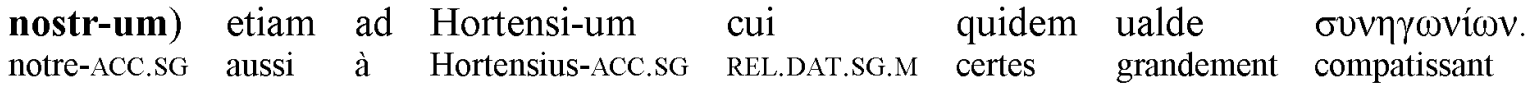

«Je t'ai écrit de Gyaros au sujet de Messala dès que j'ai appris la nouvelle; j'ai écrit aussi à Hortensius (c'est une idée que nous avons eue ensemble); je prends tout à fait part à ses ennuis. » (L.-A. Constans)

90. En effet, si nous adoptons le procédé inverse, c'est-à-dire si nous essayons de transférer à l'échelle du constituant les règles établies à l'échelle de la phrase hôte, nous pourrions supposer que la règle du placement en deuxième position est également valable à l'échelle du constituant. Or cela pourrait être le cas dans cet exemple, à condition de supposer que la conjonction et fait partie du constituant sur lequel porte l'incidente et

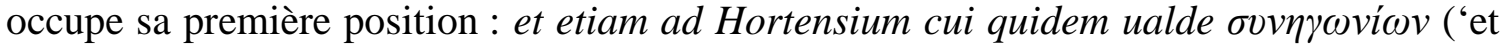
j'ai écrit aussi à Hortensius ; je prends tout à fait part à ses ennuis').

91. Notons que si l'on analyse plus en détail le domaine de portée de cette incidente, il apparaît qu'il ne s'agit pas d'un constituant nominal, mais d'une proposition coordonnée, dans laquelle le verbe est élidé, et que l'on peut reconstituer grâce à la première proposition. Dès lors, cet exemple suggèrerait que le placement en deuxième position est également possible en cas d'incidence à un constituant mais seulement si le constituant est de forme propositionnelle. De plus nombreux exemples seraient toutefois nécessaires pour valider cette affirmation.

\subsection{Bilan : le placement des incidentes}

92. Au final, il apparaît donc qu'une incidente à une phrase racine se place de manière privilégiée en deuxième position de son domaine, tandis qu'une incidente à un constituant occupe très généralement la frontière droite de son domaine. Dans le premier cas cependant, la place en fin de phrase paraît également possible. Inversement, la place en deuxième position à l'échelle du constituant paraît probable, mais plus douteuse.

93. Si l'on adopte ce schéma, on constate donc que l'apparence de hasard dans la position des incidentes n'est qu'une impression qui ne se justifie pas : au contraire, la distribution des incidentes semble finalement assez claire. Seuls deux cas se révèlent syntaxiquement ambigus : ceux de la deuxième et de la dernière position. De fait, une incidente qui occupe ces places paraît pouvoir porter aussi bien sur l'ensemble de la proposition que, respectivement, sur le premier ou le dernier constituant —elle est en effet sur le bord droit de ce groupe. Mais le sémantisme de l'incidente permet, le plus souvent, de 
trancher sans ambiguïté. On pourrait alors schématiser de la manière suivante, très simplifiée, les diverses possibilités de placement d'une incidente dans une phrase constituée de quatre groupes fonctionnels $\mathrm{G}$ (le domaine de portée est en gras), sachant que les possibilités se complexifient lorsqu' on prend en compte les sous-groupes :

- $\quad\left[\mathbf{G}^{\mathbf{1}}(\mathbf{I}) \mathbf{G}^{2} \mathbf{G}^{\mathbf{3}} \mathbf{G}^{4}\right]$ : incidente I à la proposition

- $\quad\left[\mathbf{G}^{\mathbf{1}}(\mathbf{i}) \mathrm{G}^{2} \mathrm{G}^{3} \mathrm{G}^{4}\right]$ : incidente i au premier groupe

- $\quad\left[G^{1} \mathbf{G}^{2}(\mathbf{i}) \mathrm{G}^{3} \mathrm{G}^{4}\right]$ : incidente $\mathrm{i}$ au deuxième groupe

- $\quad\left[G^{1} G^{2} G^{3}(i) G^{4}\right]$ : incidente i au troisième groupe

- $\left[G^{1} G^{2} G^{3} G^{4}(i)\right]$ : incidente i au quatrième groupe

- $\quad\left[\mathbf{G}^{1} \mathbf{G}^{2} \mathbf{G}^{3} \mathbf{G}^{\mathbf{4}}(\mathbf{I})\right]$ : incidente I à la proposition

94. En outre, on peut noter que ces contraintes distributionnelles permettent la coexistence de plusieurs incidentes dans une même phrase hôte, que l'on pourrait schématiser ainsi :

95.

$$
\left[\mathrm{G}^{1} \mathrm{G}^{2} \mathrm{G}^{3}\left(\mathrm{i}_{\mathrm{a}}\right) \mathrm{G}^{4}\left(\mathrm{i}_{\mathrm{b}}\right)\right]
$$

96. Il faut enfin préciser, comme on a pu le constater, que les procédés de relation endophorique sont à distinguer des phénomènes de portée et de domaine, ce que l'on pourrait par exemple noter ainsi :

97.

$$
\left[\mathrm{G}^{1} \mathbf{G}^{2}\left(\mathbf{i}_{\mathbf{c}}\right) \mathrm{G}_{\mathrm{c}}^{3} \mathrm{G}^{4}\right]
$$

98. où $c$ est un indice de coréférence indiquant que $\mathrm{i}$ et $\mathrm{G}$ contiennent des termes en relation endophorique.

99. Telles sont donc les règles de placement des incidentes qui semblent être pertinentes en latin. Il reste maintenant à voir si l'on peut étendre ces généralisations à d'autres langues, voire élaborer un système universel des incidentes.

Tous mes remerciements à Madame Michèle Fruyt, Monsieur Bernard Bortolussi et Madame Lyliane Sznajder pour leurs conseils. Merci également aux relecteurs anonymes de cet article.

\section{Bibliographie}

BASSARAK, Armin, 1985. Zu den Beziehungen zwischen Parenthesen und ihren Trägersätzen. Zeitschrift für Phonetik, Sprachwissenschaft und Kommunikationsforschung 38/4 : 409-417.

Betten, Anne, 1976. Ellipsen, Anakoluthe und Parenthesen. Deutsche Sprache 4 : 207230.

BolKesteIn, Alide Machtelt, 1995. Modalizing one's Message in Latin : "Parenthetical" Verbal Sentiendi. Ch.-M. Ternes \& D. Longrée (eds.) : Oratio soluta-oratio numerosa : les mécanismes linguistiques de cohésion et de rupture dans la prose latine. Actes des 8èmes rencontres scientifiques de Luxembourg, Etudes Luxembourgeoises d'Histoire et de Littérature Romaines 1 : 22-33.

Bolkestein, A. Machtelt, 1998. Between Brackets : (some Properties of) Parenthetical Clauses in Latin : an Investigation of the Language of Cicero's letters. Risselada, R. (ed.) : Latin in use : Amsterdam studies in the pragmatics of Latin, Amsterdam Studies in Classical Philology $8: 1-17$. 
Burton-Roberts, Noel, 1999. Language, Linear Precedence and Parentheticals. Peter C. Collins \& David A. Lee (ed.): The clause in English: in honour of Rodney D. Huddleston. Amsterdam : Benjamins : 33-52.

DE CORNULIER, Benoît, 1973. Les incises en français contemporain, thèse de troisième cycle, Université de Provence.

De CoRnulier, Benoît, 2001. Signification par reproduction et "incises". Studia Universitatis Babeş-Bolyai - Series philologica 46/3 : 141-143.

DELAIS-ROUSSARIE, Elisabeth, 2005. Vers une grammaire prosodique formelle : Le cas des incidentes en français. Actes électroniques de la conférence Interface Discours et Prosodie 05, Université de Provence.

Delomier, D. \& Morel, M.-A., 1986. Caractéristiques intonatives et syntaxiques des incises. DRLAV : revue de linguistique 34-35 : 141-160.

DESSAINTES, Maurice, 1960. La construction par insertion incidente. Etude grammaticale et linguistique. Paris : D'Artrey.

DE VRIES, Mark, projet de recherche 2005-2010. The Syntax of Nonsubordination: Parentheses, Appositions and Grafts, University of Groningen, téléchargeable sur : http://odur.let.rug.nl/ dvries/pdf/projectvoorstel-website.pdf.

Emonds, Joseph, 1974. Parenthetical Clauses. C. Rohrer \& N. Ruwet (ed.) : Actes du colloque franco-allemand de grammaire transformationnelle. Tübingen : Niemeyer: 192-205.

Espinal, M. Teresa, 1991. The Representation of Disjunct Constituents. Language 67-4 : 726-762.

FORGET, D., 2000. Les insertions parenthétiques. Revue québecoise de linguistique 28/2 : $15-28$.

GAPANY, J. \& Zay, F., 1995. Les relatives parenthétiques : problèmes de reconnaissance et de délimitation. Scolia $5: 31-64$.

HAND, Michael, 1993. Parataxis and Parentheticals. Linguistics and philosophy 16/5 : 495507.

HAÜSLER, Sabine, 2000. Parenthesen im Lateinischen am Beispiel der Pliniusbriefe. Glotta $76: 202-231$.

HofFMANN, Ludger, 1998. Parenthesen. Linguistische Berichte 175 : 299-329.

HofmanN, Johann Baptist, \& Szantyr, Anton, 1972 [1965]. Lateinische Grammatik 2. München : Beck.

IFANTIDOU, Elly, 1993. Parentheticals and Relevance. UCL working papers in linguistics, $5: 193-210$.

KIECKERS, Ernst, 1913. Zu den Schaltesätzen im Lateinischen, Romanischen und Neuhochdeutschen. Indogermanische Forschungen 32 : 7-23.

Kroon, Caroline, 1995. Discourse Particles in Latin : a study of nam, enim, autem, uero and at. Amsterdam : Gieben.

KÜHNER, Raphael, \& Stegmann, Carl. 1912-1914. Ausführliche Grammatik der lateinischen Sprache 2. Hanovre : Hahn.

LAMPERT, Martina, 1992. Die parenthetische Konstruktion als textuelle Strategie : zur kognitiven und kommunikativen Basis einer grammatischen Kategorie. München: Sagner.

LeHMAnN, Christian, 1989. Latin Subordination in Typological Perspective in Subordination and Other Topics in Latin, Congrès international de linguistique latine, 
Bologne, 1-5 April 1985 ; ed. Gualtiero Calboli, Studies in language companion series 17. Amsterdam : J. Benjamins : 153-179.

MCCAWLEY, James D., 1982. Parentheticals and Discontinuous Constituent Structure. Linguistic Inquiry 13 : 91-106.

MARANDIN, Jean-Marie, 1999. Grammaire de l'incidence, ms. téléchargeable : http://www.llf.cnrs.fr/Gens/Marandin/GrammaireDeL_incidence.doc.

NøLKE, Henning, 1996. Une parenthèse sur les incises. Un cas de non-intégration et de non-dépendance syntaxique. In Claude Muller (ed.), Dépendance et intégration syntaxique, Linguistische Arbeiten 351. Tübingen : Niemeyer : 317-325.

Petillon-Boucheron, Sabine, 2002. Les détours de la langue : étude sur la parenthèse et le tiret double. Louvain-Paris : Peeters.

PINKSTER, Harm, 1988. Lateinische Syntax und Semantik. Tübingen : Francke.

PITTNER, Karin, 1995. Zur Syntax von Parenthesen. Linguistische Berichte 156 : 85-108.

RoschatT, Alois, 1883. Über den Gebrauch der Parenthese in Ciceros Reden und rhetorischen Schriften. Acta seminarii philologici Erlangensis 3 : 189-244.

Roulet, Eddy, 2003. Positions of Parentheticals and Interjections : a Corpus-based Approach. In P. Fikkert \& L. Cornips (eds.), Linguistics in the Netherlands. Amsterdam : John Benjamins : 155-166.

SCHWYZER, Eduard, 1939. Die Parenthese im engeren und weiteren Sinne. Abhandlungen der Preussischen Akademie der Wissenschaften - Philosophisch-historische Klasse, 6. Berlin : de Gruyter.

Stowell, Tim, 2005. Appositive and Parenthetical Relative Clauses. In Hans Broekhuis, Norbert Corver, Riny Huybregts, Ursula Kleinherz \& Jan Koster (eds.), Organizing Grammar ; Studies in Honor of Henk van Riemsdijk. Leipzig : Mouton de Gruyter : 608617.

TOURATIER, Christian, 1994. Syntaxe latine. Louvain-la-Neuve : Peeters. 